\title{
Managing grains and interfaces via ligand anchoring enables $22.3 \%$ certified- efficiency inverted perovskite solar cells
}

Xiaopeng Zheng ${ }^{1, \dagger}$, Yi $\mathrm{Hou}^{2, \dagger}$, Chunxiong $\mathrm{Bao}^{3}$, Jun Yin ${ }^{1}$, Fanglong Yuan ${ }^{2,4}$, Ziru Huang ${ }^{2}$, Kepeng Song $^{1}$, Jiakai Liu ${ }^{1}$, Joel Troughton ${ }^{1}$, Nicola Gasparini ${ }^{1}$, Chun Zhou ${ }^{2}$, Yuanbao Lin ${ }^{1}$, Dingjiang $\mathrm{Xue}^{2}$, Bin Chen ${ }^{2}$, Andrew K. Johnston ${ }^{2}$, Nini Wei ${ }^{5}$, Mohamed Nejib Hedhili ${ }^{5}$, Mingyang Wei ${ }^{2}$, Abdullah Y. Alsalloum ${ }^{1}$, Partha Maity ${ }^{1}$, Bekir Turedi ${ }^{1}$, Chen Yang ${ }^{1}$, Derya Baran ${ }^{1}$, Thomas D. Anthopoulos ${ }^{1}$, Yu Han ${ }^{1}$, Zheng-Hong Lu ${ }^{4}$, Omar F. Mohammed ${ }^{1}$, Feng Gao ${ }^{3}$, Edward H. Sargent ${ }^{2}$, *, and Osman M. Bakr ${ }^{1, *}$

${ }^{1}$ Division of Physical Sciences and Engineering, King Abdullah University of Science and Technology (KAUST), Thuwal 23955-6900, Kingdom of Saudi Arabia.

${ }^{2}$ Department of Electrical and Computer Engineering, University of Toronto, 35 St George Street, Toronto, Ontario, M5S 3G4, Canada.

${ }^{3}$ Department of Physics, Chemistry and Biology (IFM), Linköping University, Linköping, Sweden.

${ }^{4}$ Department of Materials Science and Engineering, University of Toronto, 184 College Street, Toronto, Ontario M5S 3E4, Canada.

${ }^{5}$ Imaging and Characterization Core Lab, King Abdullah University of Science and Technology (KAUST), Thuwal 23955-6900, Saudi Arabia.

*Correspondence to: ted.sargent@utoronto.ca (E.H.S.); osman.bakr@kaust.edu.sa (O.M.B.).

†These authors contributed equally to this work. 
Inverted perovskite solar cells (PSCs) have attracted increasing attention, including because they have achieved long operating lifetimes ( $<5 \%$ efficiency loss following 1800 hours at the maximum power point (MPP) at $7^{\circ} \mathrm{C}$ ). However, inverted PSCs have until now exhibited significantly inferior power conversion efficiencies (PCEs) compared to regular PSCs. Here we reduce this efficiency gap through the use of a trace amount of surface-anchoring, longchain alkylamine ligands (AALs) as grain and interface modifiers. These, we show, suppress nonradiative carrier recombination and improve the optoelectronic properties of perovskite films. The resulting AAL surface-modified films exhibit a prominent (100) orientation and lower trap-state density, as well as enhanced carrier mobilities and diffusion lengths. These enable us to report a certified-stabilized-PCE of $22.34 \%$ (23\% PCE for lab-measured champion devices) - a record for inverted PSCs. The devices operate for over 1000 hours at the MPP, under simulated AM1.5 illumination, without loss of efficiency.

Metal halide perovskite semiconductors have seen rapid progress in their optoelectronic applications due to their broadly tunable compositions achieved using simple processing approaches ${ }^{1-5}$; and in light of their strong light absorption ${ }^{6-9}$, high charge mobility ${ }^{10,11}$, and long carrier diffusion lengths ${ }^{12-14}$. In photovoltaics (PVs), the power conversion efficiency (PCE) of single-junction perovskite solar cells (PSCs) started at 3.8\% in 2009 and has now reached 25.2\% 15 .

The highest-efficiency devices use the regular $(n-i-p)$ structure. However, removing ionicallydoped hole transport materials (HTMs) in inverted ( $p-i-n)$ devices has contributed to recent advances in device operating stability ${ }^{16}$. Recently, Bai et al. reported long-term operationally- 
stable inverted PSCs with PCE loss of less than 5\% under continuous simulated AM 1.5 irradiation for more than 1800 hours at 70 to $75^{\circ} \mathrm{C}^{17}$. Yang et al. reported inverted PSCs that maintained $97 \%$ of initial efficiency after operation at MPP under simulated AM 1.5 irradiation for 1200 hours at $65^{\circ} \mathrm{C}^{18}$. Unfortunately, the PCEs of inverted PSCs lag significantly behind those of regular structured devices $(20.9 \% \text { vs. } 25.2 \% \text { certified records })^{15,19-21}$. Photovoltage loss due to nonradiative recombination of photogenerated charge-carriers is a key factor in the inferior PCEs of inverted PSCs, despite various attempts at addressing this issue ${ }^{20,22}$.

The photovoltage of a solar cell depends on the quasi-Fermi levels of its photoactive layer, namely $E_{F n}$ and $E_{F p}$, splitting. This splitting is a function of the steady-state charge density and the bandgap of the absorber. Nonradiative carrier recombination impairs charge density buildup and diminishes the device's photovoltage. Electronic trap states caused by crystallographic defects including point defects or higher dimensional defects (such as grain boundaries (GBs)) are sources of non-radiative charge carrier recombination; numerous studies have pointed to the critical roles of point defectand GB-passivation towards achieving high-efficiency PSCs 19, 23-34. These defect sites not only militate against a high photovoltage, but also shorten the operational lifetime of perovskite devices, as they present a vulnerable initiation site for degradation by extrinsic environmental species ${ }^{35-38}$.

Here we demonstrate the use of surface-anchoring, long alkylamine ligands (AALs) - with optimized alkyl-chain length - as grain and interface modifiers to improve optoelectronic properties by promoting favorable grain orientation and suppressing trap state density. These augmented film properties enable the demonstration of a record certified PCE of $>22.3 \%(23 \%$ PCE for lab-measured champion devices) for inverted structured devices. The devices show no 
PCE loss after 1000 hours of operation at the maximum power point (MPP) under simulated AM1.5 illumination.

\section{Characterization of perovskite films}

AALs have a long alkyl hydrophobic chain as well as an amine group capable of anchoring to the A-site of perovskites. Molecules with an amine group have been implicated in promoting specific facets for perovskite films and single crystals ${ }^{39,40}$, and also filling point defects (A-site vacancies can potentially be filled by amine groups $)^{41,42}$. After the self-assembly of AALs in the perovskite matrix, the molecular interfaces are composed of densely packed ligands that are stabilized by van der Waals interactions between hydrophobic organic moieties of the ligands, and the van der Waals interactions become stronger with increasing alkyl chain length ${ }^{43}$. This quantum tunnelling layer on the surfaces and grain boundaries suppresses charge carrier nonradiative recombination ${ }^{26,43-46}$, and performs as an ion migration barrier ${ }^{47,48}$. The alkyl chain length influences ligand packing density, ligand desorption energy, carrier dynamics, and ion migration, and eventually impacts the device efficiency and stability ${ }^{43,}{ }^{48-53}$. Therefore, as a proof-of-concept, we first studied the influence of AALs ( $n$-butylamine (BA), phenethylamine (PEA), octylamine (OA), and oleylamine (OAm) with different alkyl chain lengths on the perovskite film quality.

In contrast to procedures that are meant to generate 2D/3D perovskite heterostructure, we only introduced a trace amount of AALs $<0.3 \mathrm{~mol} \%$ (note: over $5 \mathrm{~mol} \%$ large A-site cations is typically required to form 2D-3D heterostructures $)^{26,28,54,55}$. $\mathrm{Cs}_{0.05}\left(\mathrm{FA}_{0.92} \mathrm{MA}_{0.08}\right)_{0.95} \mathrm{~Pb}\left(\mathrm{I}_{0.92} \mathrm{Br}_{0.08}\right)_{3}$ (referred to as CsFAMA-8) was used as the baseline perovskite composition. The AALs (0.1 wt $\%)$ 
were directly added into the perovskite precursor and the perovskite films were fabricated using a one-step anti-solvent crystallization approach ${ }^{56}$.

We first used time-resolved photoluminescence (TRPL; Fig. 1f) to roughly assess the perovskite films' quality after introducing AALs with different alkyl chain lengths. The perovskite films with long alkyl chain AALs (OA or OAm) exhibit carrier lifetimes ( $\tau)(\mathrm{OA}: \tau=789 \mathrm{~ns}$; OAm: $\tau=$ $1049 \mathrm{~ns})$ that are much longer than pristine films ( $\tau=114 \mathrm{~ns})$ and the films with short alkyl chain AALs (BA: $\tau=195 \mathrm{~ns}$; or PEA: $\tau=265 \mathrm{~ns}$ ). This indicates that the carrier nonradiative recombination was significantly suppressed upon the introduction of a trace amount of long alkyl chain AALs.

Figure 1a-e show the water contact angles of perovskite films with different alkyl chain length AALs $(0.1 \mathrm{wt} \%)$. The water contact angle for the pristine film is $60^{\circ}$, and increased to $63^{\circ}$ and $71^{\circ}$ for the perovskite films with BA and PEA, respectively, which are short alkyl chain AALs. In contrast, the perovskite films with long alkyl chain AALs exhibited much larger water contact angles: $88^{\circ}$ and $98^{\circ}$ for the perovskite films with OA and OAm, respectively. This observation indicates that the long alkyl chain AALs assembled on perovskite film surface, thus dramatically enhancing the hydrophobicity of the films. The water contact angles (Supplementary Fig. 1) for the perovskite films with $0.05 \mathrm{wt} \%$ and the films with $0.2 \mathrm{wt} \%$ AALs are $97^{\circ}$ and $100^{\circ}$, respectively, suggesting a similar coverage of anchoring AAL molecules after anti-solvent washing.

The distinct improvement of film quality motivated us to investigate the unique role of the trace amount of long alkyl chain AALs. For the rest of the discussion, AAL refers to OAm unless 
otherwise noted. Figure 1i, $\mathbf{j}$ show scanning electron micrographs (SEMs) of CsFAMA-8 films without and with the introduction of AALs. The films with AALs show a similar grain size to that of pristine films. The SEM cross-section (Fig. 1k) shows a 750-nm-thick, highly crystalline perovskite absorber layer with a single grain throughout the thickness of the film. The powder Xray diffraction (XRD; Fig. 11) patterns show a notably increased ratio of the (100) to the other crystal plane peaks ((110), (111), (210), and (211)) in the films with AALs, which indicates that the (100) grains grew faster by consuming neighboring randomly-oriented grains. Since powder XRD conveys information primarily from the film's bulk, we also used grazing-incidence wideangle X-ray scattering (GIWAXS; Fig. 1g, h and Supplementary Fig. 2) to study the surface of the perovskite films. GIWAXS analysis of the films confirmed that the promotion of (100) occurred while other random orientations were suppressed and, moreover, no signal that could be attributable to a $2 \mathrm{D}$ component was detected. In reported 2D-3D perovskites, $2 \mathrm{D}$ perovskite platelets can be observed in SEM image and a clearly detectable 2D component signal appears in GIWAXS ${ }^{26,57}$. However, we did not observe evidence of a 2D component formation from either SEM and GIWAXS upon introduction of trace amounts of AALs, which indicates that 2D perovskite are effectively absent from our films.

The absorption of the CsFAMA-8 films is unaltered by the introduction of AALs (Fig. 1m). The Tauc-plot (Fig. 1m inset) from the UV-Vis absorption spectra shows an optical bandgap of 1.56 eV for CsFAMA-8 films. The steady photoluminescence (PL; Supplementary Fig. 3) intensity was significantly increased for films with AALs, indicating that the population of non-radiative recombination centers in the perovskite films were reduced. PL mapping confirmed the uniformly enhanced PL intensity as shown in Supplementary Fig. 4. 
We hypothesized that a suppressed trap state density and promoted (100) grain orientation resultant from the use of AALs may influence carrier transport in the perovskite films. We conducted contactless measurements of the photocarrier transport properties of CsMAFA-8 films, in which [6,6]-phenyl-C61-butyric acid methyl ester (PCBM) was used to induce the interfacial PL quenching ${ }^{58,59}$, as shown in Fig. 2a. TRPL decay measurements show that the carrier lifetime without the quencher $(\tau)$ increased from $290 \mathrm{~ns}$ for the pristine films to $520 \mathrm{~ns}$ for the films with AALs. The quench-limited diffusion time $\left(\tau_{q}\right)$ was 80 and $50 \mathrm{~ns}$ for the pristine films and the films

with AALs, respectively. From the equation ${ }^{59}: L_{D} \approx \frac{2 L}{\pi} \sqrt{2\left(\frac{\tau}{\tau_{q}}-1\right)}$, where $L_{D}$ and $L$ stand for diffusion length and film thickness $(\sim 750 \mathrm{~nm})$, respectively, we estimate a diffusion length of $\sim 1$ $\mu \mathrm{m}$ and $\sim 2 \mu \mathrm{m}$ for pristine film and the film with AALs, respectively. Combining the relation $L_{D}=$ $\sqrt{D \times \tau}$, and the Einstein relation $D=\mu \cdot k_{B} \cdot T / q$, where $\mu, k_{B}, T$ and $q$ stand for mobility, Boltzmann's constant, temperature, and elementary charge, respectively, we extracted a carrier mobility of 1.6 and $3 \mathrm{~cm}^{2} \mathrm{~V}^{-1} \mathrm{~s}^{-1}$ for pristine films and films with AALs, respectively. Thus, CsMAFA-8 films containing AALs exhibited noticeably longer diffusion length and higher carrier mobility than pristine films.

\section{Photovoltaic device architecture and performance}

The improved carrier transport in AALs treated films motivated us to investigate whether this enhancement in basic properties could be translated into improvements in device PCE, particularly through gains in photovoltage. We, thus, fabricated PSCs based on CsFAMA-8 films. As shown in Fig. 3a, the $p-i-n$ planar heterojunction devices were structured as indium tin oxide (ITO) glass 
substrate/poly(triaryl amine) (PTAA)/perovskite/fullerene (C60)/ 2,9-dimethyl-4,7-diphenyl-1,10phenanthroline $(\mathrm{BCP}) / \mathrm{copper}(\mathrm{Cu})$.

Fig. 3b shows the typical current density-voltage $(J-V)$ characteristics of CsFAMA-8 devices with different alkyl chain length AALs. The pristine devices show a typical PCE of $20.5 \%$ with a $J_{\mathrm{SC}}$ of $24.2 \mathrm{~mA} \mathrm{~cm}^{-2}$, a $V_{\mathrm{OC}}$ of $1.06 \mathrm{~V}$, and a FF of $80 \%$. The performance of the CsFAMA-8 devices with short alkyl chain AALs (BA or PEA) slightly improved to 20.8\% (BA) and 20.9\% (PEA), respectively, benefiting from $\sim 30 \mathrm{mV} V$ oc enhancement. The PEA concentration-dependent $J-V$ characteristics of CsFAMA-8 devices with PEA are shown in Supplementary Fig. 5. The champion device with PEA shows a $\mathrm{VOC}$ and PCE of $1.15 \mathrm{~V}$ and 21.3\%, respectively. In contrast, the devices with long alkyl chain AALs (OA or OAm) show a remarkably enhanced PCE, resulting from a strong Voc improvement up to $110 \mathrm{mV}$. The devices with OAm show PCE around 22.0\% with a $J_{\mathrm{SC}}$ of $24.1 \mathrm{~mA} \mathrm{~cm}^{-2}$, a $V_{\mathrm{OC}}$ of $1.17 \mathrm{~V}$, a FF of $78.2 \%$; The voltage deficit is defined by $E_{g} / q$ - Voc, where $E_{g}$ is the optical bandgap and $q$ is an elementary charge. Since the optical bandgap is $1.56 \mathrm{eV}$, the voltage deficit is only $0.39 \mathrm{~V}$ for long alkyl chain AAL-based devices. The PCEs of the devices with OA are similar to the devices containing OAm, confirming the advantage of using long alkyl chain AALs over the short ones. When fine-tuning the concentration of long alkyl chain AALs from $0.05 \mathrm{wt} \%$ to $0.2 \mathrm{wt} \%$, we did not observe an appreciable variation in device performance (Supplementary Fig. 6). Further increasing the AAL concentration to 0.4 wt $\%$ leads to a severe PCE drop. The champion devices with long alkyl chain AALs deliver a PCE of 23\% with a $J_{\mathrm{SC}}$ of $24.1 \mathrm{~mA} \mathrm{~cm}^{-2}$, a $V_{\mathrm{OC}}$ of $1.17 \mathrm{~V}$, and a FF of 81.6\%, as shown in Fig. 3c. The statistical PCE values from 100 devices (50 pristine devices and 50 devices with AALs) (Fig. 3e) demonstrate the reproducibility of the performance enhancement associated with the use of AALs. 
The devices with shorter alkyl chain AALs deliver inferior device PCE compared with long alkyl chain AALs, which is in accordance with the trend of film quality as deduced from TRPL.

Unencapsulated CsFAMA-8 devices with AALs were certified at an accredited laboratory (Newport PV Testing and Calibration Laboratory, USA). The PCE certification protocol uses a quasi-steady-state (QSS) $I-V$ sweep, instead of normally used forward and reverse $I-V$ scans, to ascertain devices PV characteristics. Ten voltage points were collected in the QSS measurement, with each bias voltage applied and held until the measured current stabilized (Supplementary Fig. 7). The devices show a certified-stabilized-PCE of $22.34 \%$ with a $J_{\mathrm{SC}}$ of $23.9 \mathrm{~mA} \mathrm{~cm}{ }^{-2}$, a $V_{\mathrm{OC}}$ of $1.14 \mathrm{~V}$, and a FF of $82 \%$, representing a record PCE for $p-i-n$ structured devices. The certified QSS I-V curve and external quantum efficiency (EQE) spectra are shown in Supplementary Fig. 8.

In order to identify the major factors responsible for the efficiency enhancement, we started with comparing the morphology (by top-view and cross-sectional SEM images) of the pristine perovskite films and the films with AALs (Supplementary Fig. 9). Both the pristine perovskite films and the films with AALs (OAm) are compact with similar grain sizes. The full width at half maximum (FWHM) values of (100) XRD peaks of pristine films and those of the films with AALs $(\mathrm{OAm})$ are $0.09^{\circ}$ and $0.10^{\circ}$, respectively, i.e. they are nearly identical. We can therefore rule out a major influence of perovskite grain size and crystallinity in this study.

To investigate the influence of surface passivation while avoiding effects related to perovskite film orientation, we subjected ready-formed pristine perovskite films to a post-treatment using alkyl 
chains of various lengths AALs (BA, PEA, and OAm). XRD patterns (Supplementary Fig. 10a) show that the films post-treated with AALs of different lengths had similar orientations as the pristine films. We then fabricated the devices based on these films with similar orientations.

The devices with post-treatment surface passivation enabled by AALs show higher PCE than the pristine devices; and the long-chain AALs show better passivation, something we assign to stronger hole-blocking at the interfaces between perovskite and $\mathrm{C}_{60}(\text { Supplementary Fig. 10b })^{46}$. The $V_{\mathrm{OC}}$ and PCE of the devices with long-chain AALs (OAm) post-treatment increased to 1.12 $\mathrm{V}$ and $21.1 \%$, respectively. However, in contrast to the devices fabricated by adding AALs (OAm) into the precursor ( $V_{\mathrm{OC}}$ of $1.17 \mathrm{~V}$ and PCE of $>22 \%$ ), the $V_{\mathrm{OC}}$ and PCE of devices with AAL (OAm) surface passivation are still lower, indicating that surface passivation alone does not fully explain the performance improvement, and that there is a crucial role for tuning the orientation of the perovskite films.

We conclude that the combined effect of both surface passivation and orientation tuning leads to the dramatically improved $V_{\mathrm{OC}}$ and PCE for the devices with AALs (mixed in the precursor). This result is rationalized by the facet-dependence of the surface defect density, and it highlights the crucial role of controlling the film orientation.

\section{Mechanisms underpinning efficiency enhancements}

We sought to evaluate whether changes in surface roughness had a significant role in improving the device efficiency. Atomic force microscopy (AFM) images revealed that the roughness of films was reduced from $17.5 \mathrm{~nm}$ to $12.1 \mathrm{~nm}$ by introducing AALs (Supplementary Fig. 11), which 
suggests that changes in the interfacial roughness were not a major variable affecting the device performance.

To further elucidate the trap state distributions and energies that are affected by the AAL treatment, we investigated trap state profiles using thermal admittance spectroscopy (TAS). We acquired temperature-dependent capacitance versus frequency $(C-f)$ plots to probe trap density and the energy depth of trap states (see supplementary materials). Fig. $2 \mathbf{b}$ shows trap density profiles at different temperatures, deduced from the temperature-dependent $C$ - $f$ plots (Supplementary Fig. 12). At all temperatures from 280 to $320 \mathrm{~K}$, the devices with AALs show similar trap state density at energies between 0.25 and $0.32 \mathrm{eV}$ and lower trap state density across the higher energy region from 0.32 to $0.4 \mathrm{eV}$. At room temperature $(300 \mathrm{~K})$, pristine devices show a peak trap density of 1 $\times 10^{23} \mathrm{~m}^{-3} \mathrm{eV}^{-1}$ at $0.35 \mathrm{eV}$; in contrast, the devices with AALs show a smaller peak intensity of 4.4 $\times 10^{22} \mathrm{~m}^{-3} \mathrm{eV}^{-1}$ at shallower energy $(0.32 \mathrm{eV})$. The pristine devices show as much as $\sim 4$ times higher trap state density in the deeper energy region from $0.32-0.4 \mathrm{eV}$. We further measured the electroluminescence (EL) spectra of solar cells operating as light-emitting diodes (LEDs), as shown in Supplementary Fig. 13. The devices with AALs deliver a quantum efficiency (EQEeL) of $0.65 \%$ under an injection current around $J_{\mathrm{SC}}$. In comparison, the pristine devices show an EQEEL of $0.07 \%$ under the same injection current. The $V_{\mathrm{OC}}$ improvement, $\Delta V_{\mathrm{OC}}$, estimated by $\Delta V O C=k_{B} T / q \ln \left(\mathrm{EQE}_{\mathrm{AAL}} / \mathrm{EQEPristine}\right)$, is $\sim 60 \mathrm{mV}$. To further test the carrier lifetime in fully operating devices, transient photo-voltage (TPV) decay measurements were conducted. As seen in Fig. 3d, the carrier lifetime $(\tau)$ increased from $0.67 \mu$ s to $1.5 \mu$ s after introducing AALs. Light intensity-dependent $V_{\mathrm{OC}}$ was measured and the diode ideal factor $(n)$ was derived, as shown in Supplementary Fig. 14. When purely radiative recombination is present, $n$ equals 1 . The devices 
with AALs show $n$ closer to 1 compared to the pristine devices, arising from the suppressed nonradiative recombination achieved by AALs.

AALs have the potential to suppress trap density directly by filling in A-site vacancies and modifying grains and interfaces; additionally, they have the potential to provide a similar benefit by promoting the (100) orientation of grains in perovskite films ${ }^{60}$, which may have a different susceptibility to surface-defect formation than other random orientations in the pristine perovskite films ${ }^{61,62}$. To further investigate this possibility, we studied the dependence of trap density on the surface crystallographic facets using density functional theory (DFT) calculations based on a cubic $\mathrm{FAPbI}_{3}$ model ${ }^{63,64}$. The corresponding slabs exposing the (100), (110), (111), (210), and (211) surfaces were considered. From the projected density of states (PDOS) of all the slabs, as shown in Fig. 4 and Supplementary Fig. 15, we find that the valence band maximum (VBM) and the conduction band minimum (CBM) are mainly comprised of I-5p, Pb-6s, and Pb-6p orbitals. According to these modeling results, some surfaces are less benign than others; the (110) (Fig. 4c), (111) (Fig. 4d), and (211) (Fig. 4e, f) surfaces show trap states that are more likely to adversely affect device performance. However, there are no trap states that appear for the (100) surface in either FAI-rich or $\mathrm{PbI}_{2}$-rich termination cases (Fig. 4a, b). Thus, it can be concluded that the (110), (111), and (211) surfaces would introduce additional trap states; while in the case of the (100) surface, the delocalized electronic distributions of the VBM and the CBM are retained in a similar configuration to bulk $\mathrm{FAPbI}_{3}$.

The electronic structure of AAL surface-modified films was investigated by work function (WF) and VBM measurements using ultraviolet photoelectron spectroscopy (UPS; Fig. 2c). The WF 
was determined from the secondary electron cutoff and we observed a change in WF from 4.58 to $4.41 \mathrm{eV}$ after AAL-modification. The VBMs are located at 1.1 and $1.3 \mathrm{eV}$ below the Fermi level $\left(E_{F}\right)$ for pristine films and AAL-modified films, respectively. The energy-level diagrams for the films based on the UPS and optical absorption measurement are shown in Fig. 2d. The VBM and CBM of AAL-modified films are nearly identical to pristine films, however, the WF shifts by about $170 \mathrm{meV}$ towards the vacuum level $\left(E_{V A C}\right)$, and the $E_{F}$ shifts by $200 \mathrm{meV}$ towards the CBM, indicating that the AAL-modified films become more n-type, which is likely attributable to the change of the surface termination after AAL-modification ${ }^{65}$. Perovskite films that are more n-type have a larger number of filled electron traps - which are the predominant trap species ${ }^{20,66}-$ and this can lead to a further decrease in trap-assisted recombination events ${ }^{20}$. Moreover, perovskite films that are more n-type enable more efficient charge transfer between the perovskite and the electron transport material (ETM; $\left.\mathrm{C}_{60}\right)^{65,67}$.

We summarized the likely mechanisms behind the device efficiency enhancement in Supplementary Fig. 16. The long chain AALs assemble on the perovskite grains, restricting the grains' tilt during the growth and eventually resulting in a (100)-orientation dominant film that is of lower defect density than a randomly oriented film (Supplementary Fig. 16a, b). As the growth proceeds, the long chain AALs are finally expelled to the perovskite film surface, as indicated by water contact-angle measurements. The ligand layer on the perovskite film's surface provides effective surface passivation because a long chain AAL layer enables strong hole-blocking between the perovskite and $\mathrm{C}_{60}$ interfaces (Supplementary Fig. 16c, d) ${ }^{46}$.

\section{Stability of devices}


Suppressing defects, and molecular modification on perovskite grains and interfaces may have the additional effect of reducing ion migration - an unwanted effect in PSCs that contributes to hysteresis and low stability. We studied ion migration using temperature-dependent transient response measurements ${ }^{68,69}$ (see supplementary materials). The ions were driven by an external bias to the electrode interface; after which this external bias was abruptly removed. A negative current can be seen due to the quick backward movement of ion vacancies forced by the concentration gradient (Supplementary Fig. 17). The decay rate $\left(\tau^{-1}\right)$ of the negative current reflects ion distribution recovery time and it was used to deduce the activation energy $\left(E_{a}\right)$ of ion migration according to the following equation:

$$
\operatorname{Ln}\left(T \tau^{-1}\right)=C-\frac{E_{a}}{k T}
$$

where $T$ is the temperature, $C$ is a constant, $k$ is the Boltzmann constant.

The typical temperature-dependent transient response measurements for CsFAMA-8 devices with AALs are shown in Supplementary Fig. 18. By fitting $\ln \left(T \tau^{-1}\right)$ vs $1 / T$, we estimate an $E_{a}$ of 243 $\mathrm{meV}$ and $422 \mathrm{meV}$ for pristine films and the films with AALs, respectively (Fig. 5a), indicating that ion migration is more hindered in the films with AALs.

Having shown that processing perovskite films with AALs leads to superior transport properties, and inverted PSCs with record PCEs, we sought to ascertain whether this processing strategy might compromise the operational stability of devices. To study the long-term stability of the perovskite photoactive layer, we conducted MPP tracking on encapsulated CsMAFA-8 devices under constant simulated AM1.5 illumination $\left(100 \mathrm{~mW} \mathrm{~cm}^{-2}\right)$, as shown in Fig. 5c. Pristine devices rapidly lost $42 \%$ of their initial PCE after 350 hours of testing, while the devices with AALs exhibited no PCE loss after continuous operation for 1000 hours under AM1.5 illumination. The 
starting device PCE was $21.2 \%$ and the final PCE after the MPP test was $21.6 \%$, which is among the highest PCEs following 1000 hours of operation at MPP. We also investigated the thermal stability by collecting $J-V$ curves at various times for devices maintained at a fixed temperature of $85^{\circ} \mathrm{C}$. The pristine devices lost $\sim 39 \%$ of their initial PCE; while the devices with AALs only lost around $10 \%$ of their initial PCE after $\sim 1020$ hours thermal stability test (Fig. 5b). A reduction in defect density and ion migration likely benefited device stability ${ }^{18,22}$.

\section{Conclusion}

In summary, we have demonstrated the novel role of AALs, particularly long-alkyl chain AALs, in improving optoelectronic properties by promoting favorable grain orientations and suppressing trap state density. Our long alkyl-chain AALs-based strategy enabled us to demonstrate $>23.0 \%$ efficient (22.3\% certified) $p-i-n$ structured devices and good operational stability. The molecular modification of perovskite grains and interfaces is crucial to enhance both the efficiency and stability of PSCs. Our findings also indicated that anisotropic electronic properties of the perovskite facets make management of films' crystal orientations an important path towards highefficiency PSCs. Further understanding of the nature of the different surface facets in polycrystalline perovskite, including the defect type and concentration, will likely be essential for the future of perovskite devices.

\section{Methods}

Perovskite film preparation and device fabrication. The patterned ITO/glass substrates were sequentially cleaned with soap, deionized water, acetone, and isopropanol under ultrasonication. The ITO/glass substrates were then dried with $\mathrm{N}_{2}$ blow and treated with ultraviolet ozone for 
$15 \mathrm{~min}$. The perovskite films were fabricated by the anti-solvent crystallization approach in $\mathrm{N}_{2}$ glove box. In a typical procedure, a hole transport layer (HTL) poly(bis(4-phenyl)(2,4,6trimethylphenyl)amine) (PTAA) with a concentration of $2 \mathrm{mg} / \mathrm{ml}$ dissolved in toluene were spin coated at the speed of 6,000 r.p.m for $35 \mathrm{~s}$ and then annealed at $100{ }^{\circ} \mathrm{C}$ for $15 \mathrm{~min}$. The perovskite precursor solution (1.4 M) composed of mixed cations (lead (Pb), cesium(Cs), formamidinium (FA) and methylammonium (MA)) and halides (I, Br) was dissolved in mixed solvent $(\mathrm{DMF} / \mathrm{DMSO}=4: 1)$ according to a formula of $\mathrm{Cs}_{0.05}\left(\mathrm{FA}_{0.92} \mathrm{MA}_{0.08}\right)_{0.95} \mathrm{~Pb}\left(\mathrm{I}_{0.92} \mathrm{Br}_{0.08}\right)_{3}$. A trace amount of alkylammonium ligands was added into the precursor solution. A two-step spin-coating procedure with $2000 \mathrm{rpm}$ for $10 \mathrm{~s}$ and $4000 \mathrm{rpm}$ for the $50 \mathrm{~s}$ was adopted for the preparation of perovskite films. Chlorobenzene $(\mathrm{CB} ; 150 \mu \mathrm{L})$ was dropped on the spinning substrate when the 45 s of second spin coating step. Subsequently, the sample was annealed at $100{ }^{\circ} \mathrm{C}$ for $30 \mathrm{~min}$. The devices with area of $10 \mathrm{~mm}^{2}$ were finished by thermally evaporating $\mathrm{C}_{60}(20 \mathrm{~nm}), \mathrm{BCP}(3 \mathrm{~nm})$ and copper $(\mathrm{Cu} ; 80 \mathrm{~nm})$ in sequential order by using a thin film deposition system from Angstrom Engineering under high vacuum (5e-6 Torr).

Perovskite film characterizations. The SEM images were taken by a Helios G4 UX dual beam scanning electron microscope (SEM). XRD patterns were measured with a Bruker AXS D8 diffractometer using $\mathrm{Cu} \mathrm{K \alpha}$ radiation $(\lambda=1.54178 \AA$ ). The absorption spectra were obtained by Cary 6000i UV-Vis-NIR spectrophotometer. The roughness was measured by an atomic force microscope (Bruker Dimension Icon with ScanAsyst). Photoluminescence measurements were performed using a Horiba Fluorolog Time Correlated Single Photon Counting system with photomultiplier tube detectors. The excitation source is a pulsed laser diode at a wavelength of 504 $\mathrm{nm}$. The water contact angle was measured by Drop Shape Analyzer DSA100 from KRUSS with 
uniform LED illumination and high-quality optics to ensure high precision when displaying the drop for accurately measuring the contact angle. GIWAXS measurements were carried out at beamline 7.3.3 of the Advanced Light Source, Lawrence Berkeley National Laboratory. Samples were measured at a detector distance of $0.249 \mathrm{~m}$ using X-ray wavelength of $1.240 \AA$, at $0.24^{\circ}$ angle of incidence with respect to the substrate plane. Scattering intensity was detected by a PILATUS $2 \mathrm{M}$ detector.

Device characterizations. Simulated AM $1.5 \mathrm{G}$ irradiation $\left(100 \mathrm{~mW} / \mathrm{cm}^{2}\right)$ was produced by a Xenon-lamp-based solar simulator (Abet Technologies Sun 3000 Class AAA Solar Simulator) for current density-voltage $(J-V)$ measurements. The light intensity was calibrated by a calibrated reference cell with a Schott visible-color glass-filtered (KG5 color-filtered) from Newport Corporation. Keithley 2400 Source-Meter was used for driving $J$ - $V$ measurement. The devices were measured immediately after fabrication without any preconditioning. It was measured with a metal aperture with area of $6.69 \mathrm{~mm}^{2}$ (certified by Newport) to accurately define the active area. A voltage scan was measured from $1.3 \mathrm{~V}$ to $-0.3 \mathrm{~V}$ with a scanning rate of $0.1 \mathrm{~V} \mathrm{~s}^{-1}$ and a voltage step of $10 \mathrm{mV}$ was used. The devices were measured in both nitrogen atmosphere (at KAUST) and air ambient (humidity of 30-60\%; at University of Toronto) and no obvious difference was observed. To further confirm the PV parameters, the devices were measured by an independent accredited laboratory (Newport Photovoltaic Testing and Calibration Laboratory in Bozeman, Montana, U.S.). A quasi-steady-state (QSS) I-V sweep protocol was used to get rid of the influence from the hysteresis. For QSS I-V sweep conducted by Newport PV Lab, 10 voltage points were collected in QSS measurement, and each bias voltage was applied and held until the measured current was determined to be unchanging. The $J_{\mathrm{SC}}$ and PCE measured by Newport is similar to the 
result in the lab. The EQE was measured by Newport PV Lab and the integrated $J_{\mathrm{SC}}$ was carefully checked and matched well with $J_{\mathrm{SC}}$ from I-V sweep before they provide the certificate. For the transient photo-voltage (TPV) measurements, the device was serially connected to a digital oscilloscope for monitoring the charge density decay for the device in approximately $V_{\mathrm{OC}}$ condition. An attenuated a $405 \mathrm{~nm}$ laser-diode was used as a small perturbation to the background illumination on the device. The laser-pulse-induced photo-voltage variation $(\Delta V)$ and the $V_{\mathrm{OC}}$ is produced by the background illumination. The intensity of the short (50 ns) laser pulse was adjusted to keep the voltage perturbation below $10 \mathrm{mV}$, typically at $5 \mathrm{mV}$. A Keithley 2400 sourcemeter and a fibre integration sphere (FOIS-1) coupled with a QE Pro spectrometer (Ocean Optics) were used for the measurements of the devices as the LEDs.

Trap density measurements by thermal admittance spectroscopy (TAS). A sinusoidal voltage $\left(\mathrm{V}_{\text {peak-to-peak }}=30 \mathrm{mV}\right)$ generated from a function generator (Tektronix AFG 3000) was applied to the device. The current signal of the devices was analyzed using a lock-in amplifier (Stanford Research Systems, SR830) after amplified through a low-noise-current preamplifier (Stanford Research Systems, SR570). The capacitance of the device was calculated based on the parallel equivalent circuit model with the amplitude and phase of the current signal obtained from the lockin amplifier. The capacitance spectra of the device were measured by scanning the frequency of the sinusoidal voltage from 0.01 to $100 \mathrm{kHz}$ in logarithmic steps. The temperature of the device was controlled using a closed cycle cryocooler (Advanced Research Systems, DE202AE). The capacitance-voltage curve was obtained by measuring the capacitance as the applied D.C. the bias voltage was scanned from -0.2 to $1.5 \mathrm{~V}$. Based on the capacitance spectra measured at different temperatures, the trap density distribution in energy $(E \omega)$ was calculated. 
Ion migration measurement. To measure the ion migration activation energy, two symmetric $\mathrm{Au}$ electrodes were deposited on the perovskite films to form a symmetric Au/perovskite/Au device. The electrode width is $2 \mathrm{~mm}$ and the channel length is $50 \mu \mathrm{m}$. A bias of $3 \mathrm{~V}$ voltage was first applied to the electrodes by a source meter (Keithley 2400) for about $16 \mathrm{~s}$ to induce the ion migration. Then a negative current induced by the ion vacancies re-distribution can be measured after the voltage was suddenly removed. The negative current then will exponentially recovered to zero. The decay time of the negative current contains the information of the ionic transport dynamics. The measurement was carried out when the temperature of the device was controlled from 280 to $325 \mathrm{~K}$ using a DE202AE closed cycle cryocooler (Advanced Research Systems). Then the negative current curves were fitted with a double-exponential function. The slower decay time constants $\tau$ which represent the ion distribution recovery time were used to deduce the activation energy of the ion migration.

Computational methods. The density functional theory (DFT) calculations were performed with the projector-augmented wave (PAW) method as implemented in the VASP code. The generalized gradient approximation Perdew-Burke-Ernzerhof (PBE) exchange-correlation functional was used. A uniform grid of $6 \times 6 \times 6 k$-mesh in the Brillouin zone was employed to optimize the crystal structures of cubic-phase $\mathrm{FAPbI}_{3}$. We considered $\mathrm{FAPbI}_{3}$ slabs exposing different surfaces; all the slabs were separated by both top and bottom vacuum layers $(\sim 10 \AA)$ to prevent spurious inter-slab interactions. The Brillouin zone was sampled by a $2 \times 2 \times 1 k$-mesh for FAPbI 3 slabs. The planewave basis set cutoffs of the wavefunctions were set at $500 \mathrm{eV}$ for bulk crystals, $450 \mathrm{eV}$ for supercells at the GGA/PBE level. The atomic positions of all supercells with and without defects 
were fully relaxed until the supercells had Hellman-Feynman forces on each atom less than 0.01 $\mathrm{eV} / \AA$.

Device stability measurement. The operational stability tests were carried out at MPP for the encapsulated devices under one-sun illumination with a 420-nm cutoff UV filter. The devices were tested in a chamber with constant nitrogen supply, which also ensured a stable device temperature of $\sim 40^{\circ} \mathrm{C}$ (the chamber itself was in ambient air). The voltage at MPP was calculated and applied automatically; the current output of the device was tracked. The devices were encapsulated in a nitrogen glovebox. UV-curable epoxy was coated around the device area and a glass cover slide was attached to enclose the area. The encapsulation was then finished off by exposing the construct to UV light for 10 mins. The thermal stability assessment of solar cells was carried out by repeating the $J$ - $V$ test over various times for the devices heated at a fixed temperature of $85^{\circ} \mathrm{C}$.

Reporting Summary. Further information on research design is available in the Nature Research Reporting Summary linked to this article.

\section{Data availability}

The data that support the plots within this paper and other finding of this study are available from the corresponding author upon reasonable request.

\section{References:}

1. Kojima, A., Teshima, K., Shirai, Y. \& Miyasaka, T. Organometal halide perovskites as visible-light sensitizers for photovoltaic cells. J. Am. Chem. Soc. 131, 6050-6051 (2009).

2. Liu, Z. et al. Gas-solid reaction based over one-micrometer thick stable perovskite films for efficient solar cells and modules. Nat. Commun. 9, 3880 (2018). 
3. Jiang, Q. et al. Enhanced electron extraction using $\mathrm{SnO}_{2}$ for high-efficiency planarstructure $\mathrm{HC}\left(\mathrm{NH}_{2}\right)_{2} \mathrm{PbI}_{3}$-based perovskite solar cells. Nat. Energy 2, 16177 (2016).

4. Wu, Y. et al. Perovskite solar cells with $18.21 \%$ efficiency and area over $1 \mathrm{~cm}^{2}$ fabricated by heterojunction engineering. Nat. Energy 1, 16148 (2016).

5. Yang, M. et al. Perovskite ink with wide processing window for scalable high-efficiency solar cells. Nat. Energy 2, 17038 (2017).

6. Hou, Y. et al. A generic interface to reduce the efficiency-stability-cost gap of perovskite solar cells. Science 358, 1192-1197 (2017).

7. Tsai, H. et al. Light-induced lattice expansion leads to high-efficiency perovskite solar cells. Science 360, 67-70 (2018).

8. Zhao, D. et al. Efficient two-terminal all-perovskite tandem solar cells enabled by highquality low-bandgap absorber layers. Nat. Energy 3, 1093-1100 (2018).

9. $\mathrm{Ke}, \mathrm{W}$. et al. Enhanced photovoltaic performance and stability with a new type of hollow 3D perovskite \{en\}FASnI 3. Sci. Adv. 3, e1701293 (2017).

10. Kim, H.-S. et al. Lead iodide perovskite sensitized all-solid-state submicron thin film mesoscopic solar cell with efficiency exceeding 9\%. Sci. Rep. 2, 591 (2012).

11. Wehrenfennig, C., Eperon, G.E., Johnston, M.B., Snaith, H.J. \& Herz, L.M. High charge carrier mobilities and lifetimes in organolead trihalide perovskites. Adv. Mater. 26, 15841589 (2014).

12. Shi, D. et al. Low trap-state density and long carrier diffusion in organolead trihalide perovskite single crystals. Science 347, 519-522 (2015).

13. Dong, Q. et al. Electron-hole diffusion lengths $>175 \mu \mathrm{m}$ in solution-grown $\mathrm{CH}_{3} \mathrm{NH}_{3} \mathrm{PbI}_{3}$ single crystals. Science 347, 967-970 (2015).

14. Chen, Z. et al. Single-crystal MAPbI 3 perovskite solar cells exceeding $21 \%$ power conversion efficiency. ACS Energy Lett., 1258-1259 (2019).

15. National Renewable Energy Laboratory, best research-cell efficiencies (2019). www.nrel.gov/pv/assets/pdfs/pv-efficiency-chart.20190103.pdf.

16. Christians, J.A. et al. Tailored interfaces of unencapsulated perovskite solar cells for $>1,000$ hour operational stability. Nat. Energy 3, 68-74 (2018).

17. Bai, S. et al. Planar perovskite solar cells with long-term stability using ionic liquid additives. Nature 571, 245-250 (2019).

18. Yang, S. et al. Stabilizing halide perovskite surfaces for solar cell operation with widebandgap lead oxysalts. Science 365, 473-478 (2019).

19. Jiang, Q. et al. Surface passivation of perovskite film for efficient solar cells. Nat. Photonics 13, 460-466 (2019).

20. Luo, D. et al. Enhanced photovoltage for inverted planar heterojunction perovskite solar cells. Science 360, 1442-1446 (2018).

21. Turren-Cruz, S.-H., Hagfeldt, A. \& Saliba, M. Methylammonium-free, high-performance, and stable perovskite solar cells on a planar architecture. Science 362, 449-453 (2018).

22. Zheng, $X$. et al. Defect passivation in hybrid perovskite solar cells using quaternary ammonium halide anions and cations. Nat. Energy 2, 17102 (2017).

23. $\mathrm{Bi}$, D. et al. Polymer-templated nucleation and crystal growth of perovskite films for solar cells with efficiency greater than 21\%. Nat. Energy 1, 16142 (2016).

24. Zheng, X. et al. Quantum dots supply bulk- and surface-passivation agents for efficient and stable perovskite solar cells. Joule 3, 1963-1976 (2019). 
25. Tong, J. et al. Carrier lifetimes of $>1 \mu \mathrm{s}$ in $\mathrm{Sn}-\mathrm{Pb}$ perovskites enable efficient allperovskite tandem solar cells. Science 364, 475-479 (2019).

26. Wang, Z. et al. Efficient ambient-air-stable solar cells with 2D-3D heterostructured butylammonium-caesium-formamidinium lead halide perovskites. Nat. Energy 2, 17135 (2017).

27. Yang, W.S. et al. Iodide management in formamidinium-lead-halide-based perovskite layers for efficient solar cells. Science 356, 1376-1379 (2017).

28. Liu, Y. et al. Ultrahydrophobic 3D/2D fluoroarene bilayer-based water-resistant perovskite solar cells with efficiencies exceeding 22\%. Sci. Adv. 5, eaaw2543 (2019).

29. $\mathrm{Li}, \mathrm{X}$. et al. Improved performance and stability of perovskite solar cells by crystal crosslinking with alkylphosphonic acid $\omega$-ammonium chlorides. Nat. Chem. 7, 703 (2015).

30. Saidaminov, M.I. et al. Suppression of atomic vacancies via incorporation of isovalent small ions to increase the stability of halide perovskite solar cells in ambient air. Nat. Energy 3, 648-654 (2018).

31. Deng, Y. et al. Surfactant-controlled ink drying enables high-speed deposition of perovskite films for efficient photovoltaic modules. Nat. Energy 3, 560-566 (2018).

32. Stolterfoht, M. et al. Visualization and suppression of interfacial recombination for highefficiency large-area pin perovskite solar cells. Nat. Energy 3, 847-854 (2018).

33. Yang, D. et al. High efficiency planar-type perovskite solar cells with negligible hysteresis using EDTA-complexed $\mathrm{SnO}_{2}$. Nat. Commun. 9, 3239 (2018).

34. Wang, Y. et al. Thermodynamically stabilized $\beta-\mathrm{CsPI}_{3}-$ based perovskite solar cells with efficiencies $>18 \%$. Science 365, 591-595 (2019).

35. Li, N. et al. Cation and anion immobilization through chemical bonding enhancement with fluorides for stable halide perovskite solar cells. Nat. Energy 4, 408 (2019).

36. Wang, S., Jiang, Y., Juarez-Perez, Emilio J., Ono, Luis K. \& Qi, Y. Accelerated degradation of methylammonium lead iodide perovskites induced by exposure to iodine vapour. Nat. Energy 2, 16195 (2016).

37. Wang, Y. et al. Stabilizing heterostructures of soft perovskite semiconductors. Science 365, 687-691 (2019).

38. Chen, M. et al. Highly stable and efficient all-inorganic lead-free perovskite solar cells with native-oxide passivation. Nat. Commun. 10, 16 (2019).

39. Jao, M.-H., Lu, C.-F., Tai, P.-Y. \& Su, W.-F. Precise facet engineering of perovskite single crystals by ligand-mediated strategy. Cryst. Growth Des. 17, 5945-5952 (2017).

40. Muscarella, L.A. et al. Air-stable and oriented mixed lead halide perovskite (FA/MA) by the one-step deposition method using zinc iodide and an alkylammonium additive. ACS Appl. Mater. Interfaces 11, 17555-17562 (2019).

41. $\mathrm{Wu}, \mathrm{W} .-\mathrm{Q}$. et al. Bilateral alkylamine for suppressing charge recombination and improving stability in blade-coated perovskite solar cells. Sci. Adv. 5, eaav8925 (2019).

42. Yang, S. et al. Tailoring passivation molecular structures for extremely small open-circuit voltage loss in perovskite solar cells. J. Am. Chem. Soc. 141, 5781-5787 (2019).

43. Proppe, A.H. et al. Photochemically cross-linked quantum well ligands for $2 \mathrm{D} / 3 \mathrm{D}$ perovskite photovoltaics with improved photovoltage and stability. J. Am. Chem. Soc. 141, 14180-14189 (2019).

44. Qing, J. et al. High-quality ruddlesden-popper perovskite films based on in situ formed organic spacer cations. Adv. Mater. 0, 1904243. 
45. Fei, C. et al. Self-assembled propylammonium cations at grain boundaries and the film surface to improve the efficiency and stability of perovskite solar cells. J. Mater. Chem. A 7, 23739-23746 (2019).

46. Wang, Q., Dong, Q., Li, T., Gruverman, A. \& Huang, J. Thin insulating tunneling contacts for efficient and water-resistant perovskite solar cells. Adv. Mater. 28, 67346739 (2016).

47. Lin, Y. et al. Suppressed ion migration in low-dimensional perovskites. ACS Energy Lett. 2, 1571-1572 (2017).

48. Xiao, X. et al. Suppressed ion migration along the in-plane direction in layered perovskites. ACS Energy Lett. 3, 684-688 (2018).

49. Yoo, J.J. et al. An interface stabilized perovskite solar cell with high stabilized efficiency and low voltage loss. Energy Environ. Sci. 12, 2192-2199 (2019).

50. Zuo, L. et al. Polymer-modified halide perovskite films for efficient and stable planar heterojunction solar cells. Sci. Adv. 3, e1700106 (2017).

51. Koh, T.M. et al. Enhancing moisture tolerance in efficient hybrid 3D/2D perovskite photovoltaics. J. Mater. Chem. A 6, 2122-2128 (2018).

52. Yang, S. et al. Functionalization of perovskite thin films with moisture-tolerant molecules. Nat. Energy 1, 15016 (2016).

53. Quintero-Bermudez, R. et al. Ligand-induced surface charge density modulation generates local type-II band alignment in reduced-dimensional perovskites. J. Am. Chem. Soc. 141, 13459-13467 (2019).

54. Zhou, T. et al. Highly efficient and stable solar cells based on crystalline oriented 2D/3D hybrid perovskite. Adv. Mater. 31, 1901242 (2019).

55. Jodlowski, A.D. et al. Large guanidinium cation mixed with methylammonium in lead iodide perovskites for 19\% efficient solar cells. Nat. Energy 2, 972-979 (2017).

56. Jeon, N.J. et al. Solvent engineering for high-performance inorganic-organic hybrid perovskite solar cells. Nat. Mater. 13, 897 (2014).

57. Yang, R. et al. Oriented quasi-2D perovskites for high performance optoelectronic devices. Adv. Mater. 30, 1804771 (2018).

58. Gong, X. et al. Contactless measurements of photocarrier transport properties in perovskite single crystals. Nat. Commun. 10, 1591 (2019).

59. Lee, E.M.Y. \& Tisdale, W.A. Determination of exciton diffusion length by transient photoluminescence quenching and its application to quantum dot films. J. Phys. Chem. C 119, 9005-9015 (2015).

60. Jariwala, S. et al. Local crystal misorientation influences non-radiative recombination in halide perovskites. Joule, https://doi.org/10.1016/j.joule.2019.09.001 (2019).

61. Zhang, L. et al. Exploring anisotropy on oriented wafers of $\mathrm{MAPbBr}_{3}$ crystals grown by controlled antisolvent diffusion. Cryst. Growth Des. 18, 6652-6660 (2018).

62. Kim, D. et al. Probing facet-dependent surface defects in $\mathrm{MAPbI}_{3}$ perovskite single crystals. J. Phys. Chem. C 123, 14144-14151 (2019).

63. Kresse, G. \& Hafner, J. Ab initio molecular dynamics for open-shell transition metals. Phys. Rev. B 48, 13115-13118 (1993).

64. Kresse, G. \& Furthmüller, J. Efficient iterative schemes for ab initio total-energy calculations using a plane-wave basis set. Phys. Rev. B 54, 11169-11186 (1996). 
65. Quarti, C., De Angelis, F. \& Beljonne, D. Influence of surface termination on the energy level alignment at the $\mathrm{CH}_{3} \mathrm{NH}_{3} \mathrm{PbI}_{3}$ perovskite/C60 interface. Chem. Mater. 29, 958-968 (2017).

66. Stranks, S.D. et al. Recombination kinetics in organic-inorganic perovskites: excitons, free Charge, and subgap states. Phys. Rev. Applied 2, 034007 (2014).

67. Habisreutinger, S.N., Noel, N.K., Snaith, H.J. \& Nicholas, R.J. Investigating the role of 4-tert butylpyridine in perovskite solar cells. Adv. Energy Mater. 7, 1601079 (2017).

68. $\mathrm{Li}$, D. et al. Electronic and ionic transport dynamics in organolead halide perovskites. ACS Nano 10, 6933-6941 (2016).

69. Pan, W. et al. $\mathrm{Cs}_{2} \mathrm{AgBiBr} 6$ single-crystal $\mathrm{X}$-ray detectors with a low detection limit. Nat. Photonics 11, 726-732 (2017).

\section{Acknowledgments}

X.Z. and Y.H. contributed equally to this work. The authors acknowledge the use of KAUST Core Lab and KAUST Solar Center facilities. This work was supported by King Abdullah University of Science and Technology (KAUST), and Office of Sponsored Research (OSR) under award no. OSR-2017-CRG-3380.

\section{Author contributions}

O.M.B., X.Z., Y.H., and E.H.S. conceived the idea and designed the experiments. X.Z. fabricated the devices and conducted the characterizations. Y. H. contributed to device fabrication and evaluation, stability test, and TRPL measurement. C.B. contributed to measurements of TAS, TPV, ion migration, and LEDs. J.Y. did and interpreted the DFT calculation. J.L. did XRD measurements. A.K.J. did GIWAXS measurements. K.S., J.L., N.W., B.T., and C.Y. contributed to electron-microscopy measurements. N.G. contributed to light intensity dependent $J$-V measurements. Y.L. contributed to AFM and contact angle measurements. A.Y.A contributed to PL and PL mapping. F.Y., C.Z., Z.H., P.M., D.X., B.C., and M.W. contributed to TRPL and analyses. M.N.H. did UPS measurements. J.T. contributed to stability measurements. D.B., 
T.D.A., Y.H., Z.H.L., O.F.M., F.G., and E.H.S. provided advice and expertise. X.Z., O.M.B., Y.H., and E.H.S. composed the manuscript. All authors contributed to the revisions of the manuscript.

\section{Competing interests}

The authors declare no competing interests.

\section{Additional information}

Correspondence and requests for materials should be addressed to E.H.S. or O.M.B. 

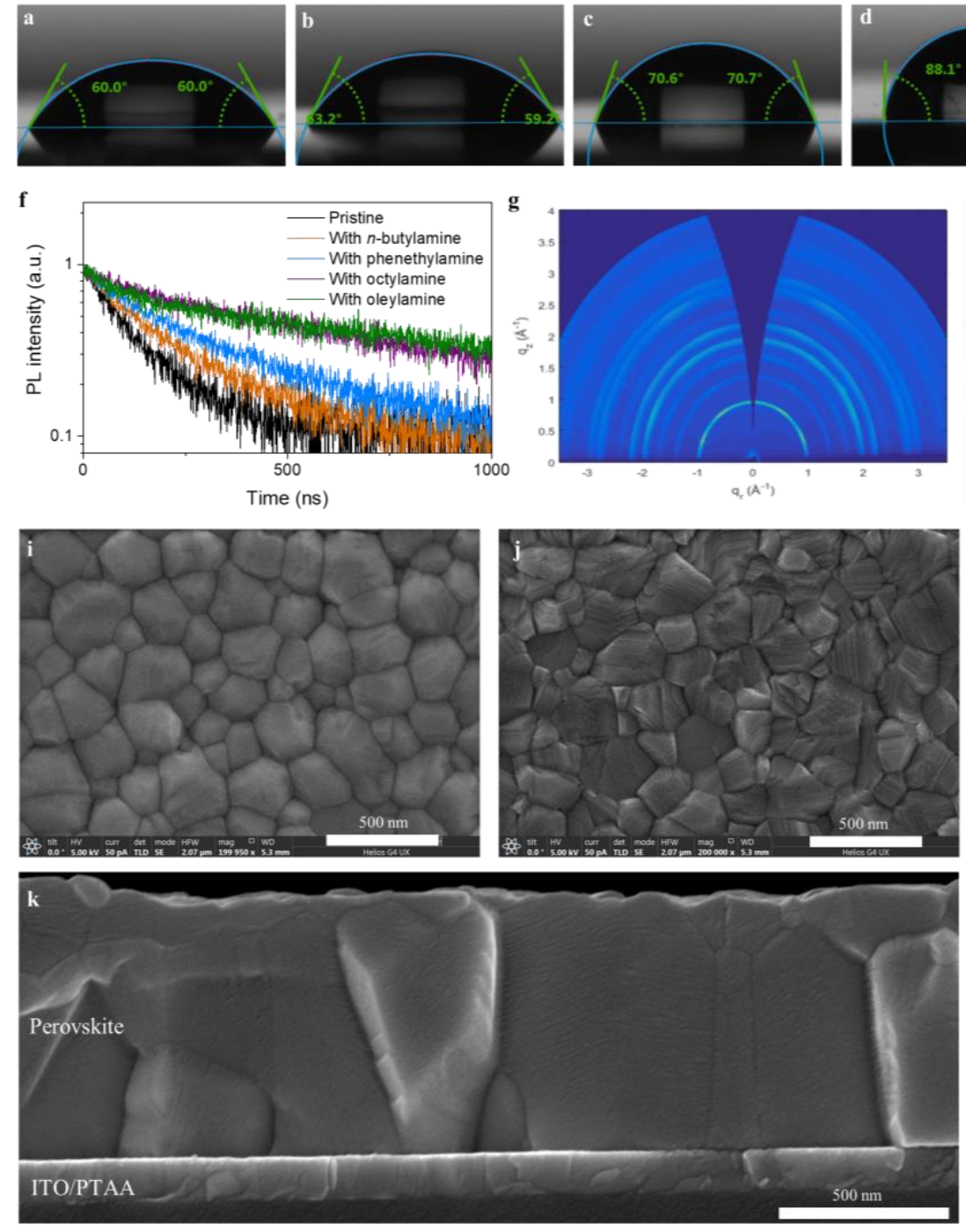
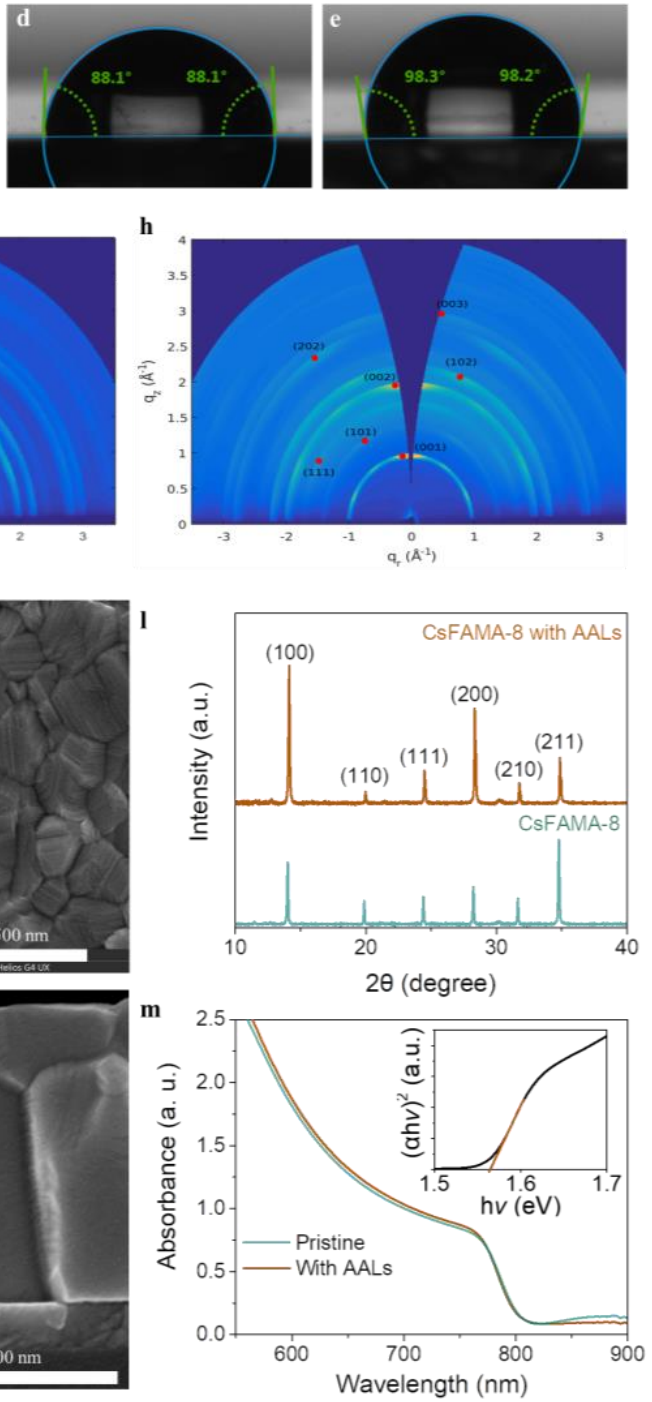

Figure 1. Characterization of perovskite films. a-e, Water contact angles of the pristine CsFAMA8 film (a) and the CsFAMA-8 film with BA (b), PEA (c), OA (d), and OAm (e).f, TRPL decays of CsFAMA-8 films with different alkyl chain length AALs. $\boldsymbol{g}$ and $\boldsymbol{h}$, GIWAXS maps of the pristine CsFAMA-8 film and the CSFAMA-8 film with AALs (OAm), respectively. $\boldsymbol{i}$ and $\boldsymbol{j}$, Top-view SEM images of the pristine CSFAMA-8 film and the CsFAMA-8 film with AALs (OAm), respectively. $\boldsymbol{k}$, Cross-sectional SEM image of the CsFAMA-8 film with AALs (OAm). l, XRD patterns of pristine CsFAMA-8 film, and CsFAMA-8 film with AALs (OAm). m, UV-Vis absorption spectra for the pristine CsFAMA-8 film and the CsFAMA-8 film with AALs (OAm). Inset is Tauc plot. 

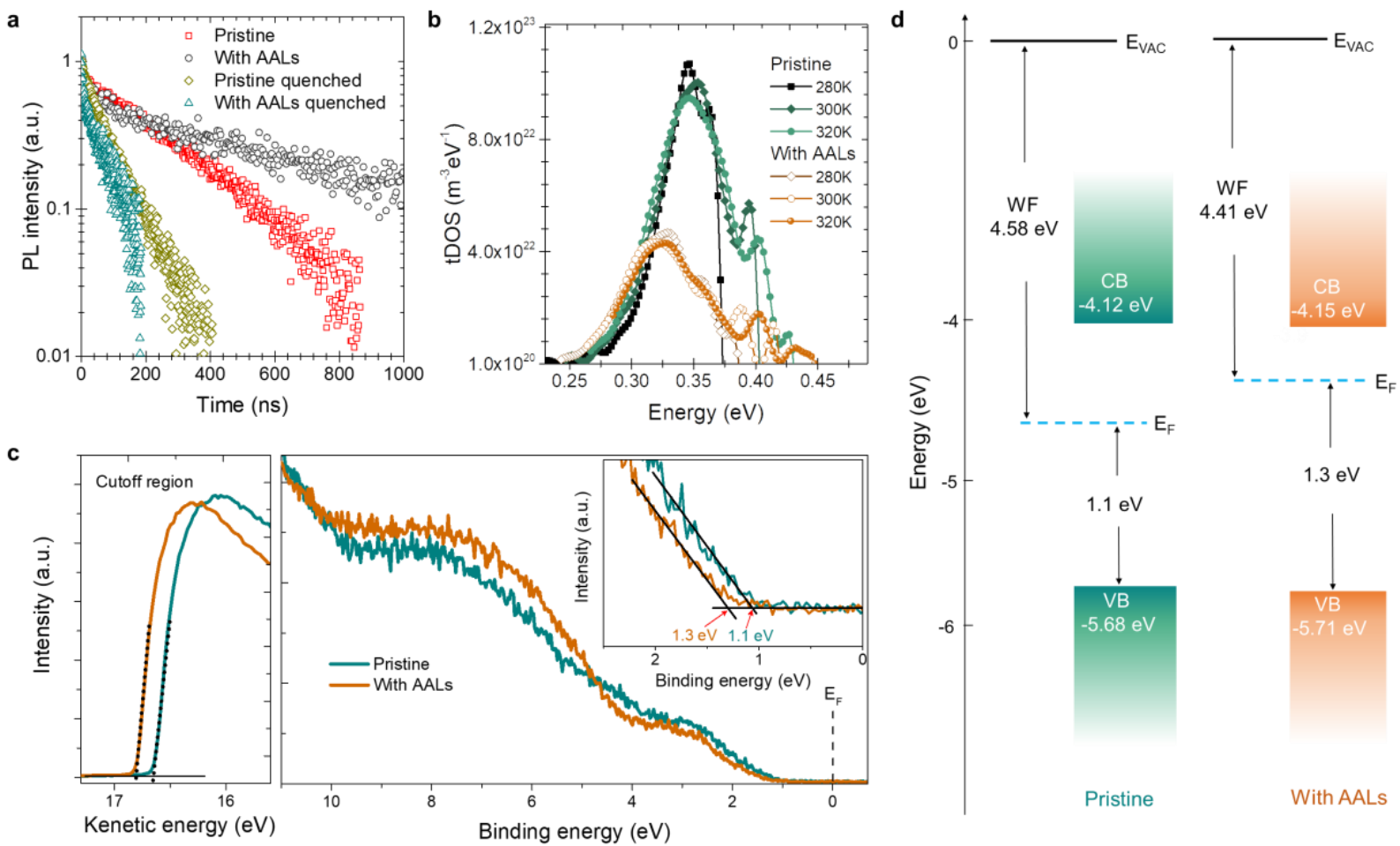

Figure 2. Time-resolved photoluminescence (TRPL), thermal admittance spectroscopy (TAS), and ultraviolet photoelectron spectroscopy (UPS)measurements. a, TRPL decay curves for the pristine CsFAMA-8 film, CsFAMA-8 film with AALs (OAm), quenched pristine film, and quenched film with AALs (OAm). $\boldsymbol{b}$, Trap state density deduced from the C-f plots for the pristine CsFAMA8 device and the CsFAMA-8 device with AALs (OAm). c, Helium I $(\mathrm{hv}=21.22 \mathrm{eV})$ spectra of secondary electron cutoff and valence band for the pristine CsFAMA-8 film and the film with AALs (OAm).d, Energy level scheme for the pristine CsFAMA-8 film and the film with AALs (OAm). 
a

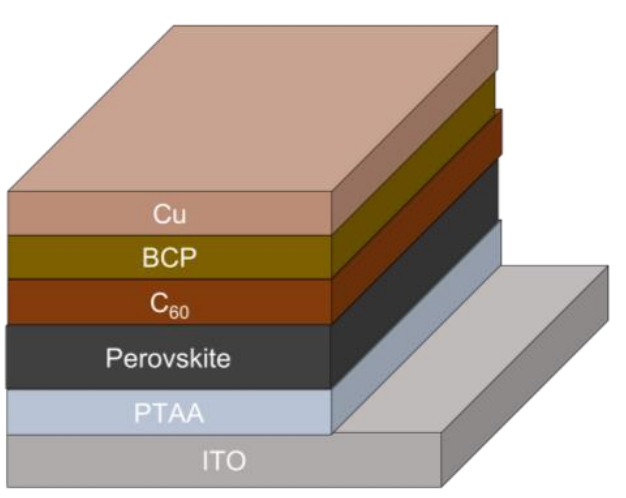

C
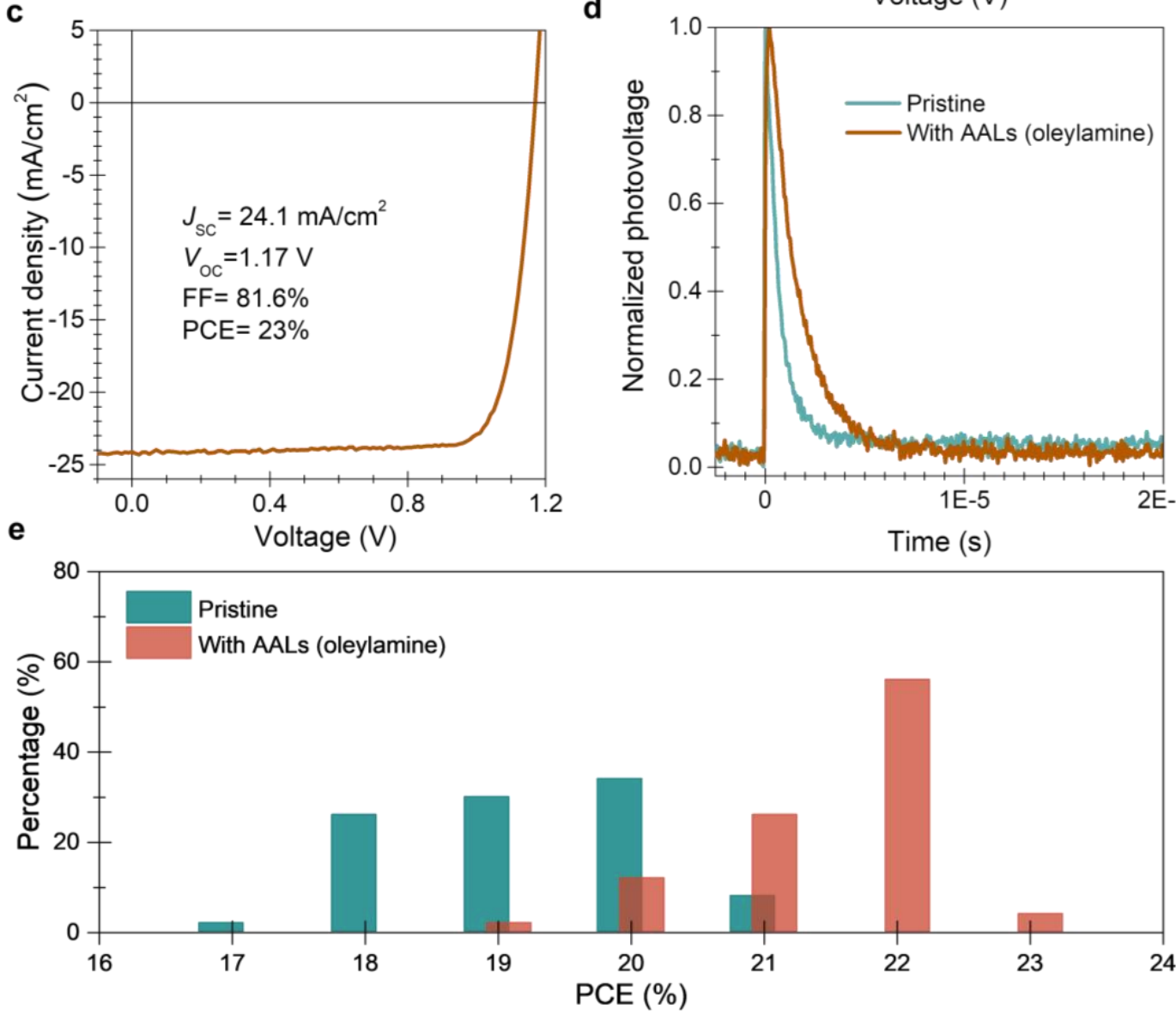

b

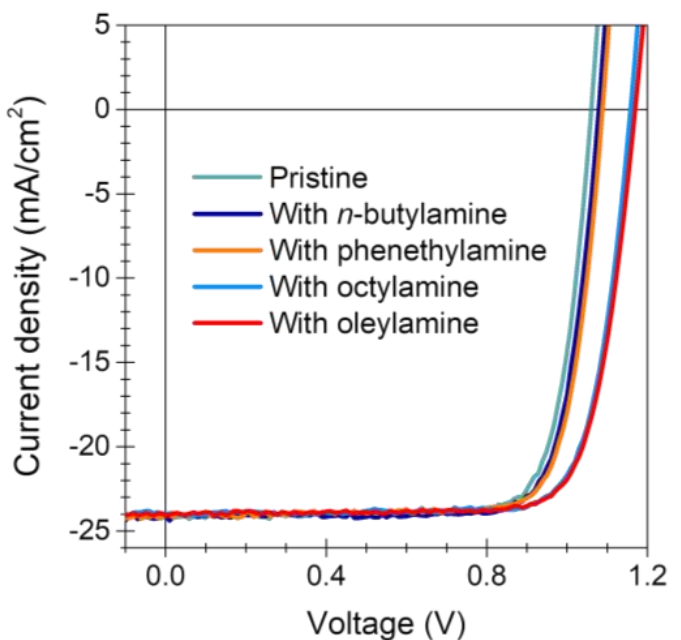

d

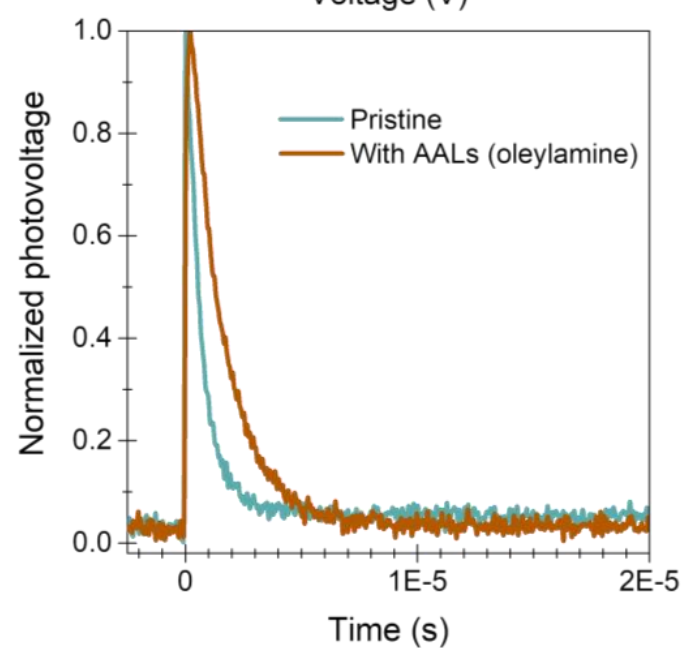

Figure 3. Device structure and photovoltaic performance analysis. a, The device architecture of

3 inverted planar heterojunction PSCs. b, Current density-voltage (J-V) characteristics of 4 CsFAMA-8 devices with addition of different alkyl chain length AALs. c, J-V curves of the 
5 champion CsFAMA-8 device with AALs (OAm).d, Transient photovoltage (TPV) measurement of

6 CsFAMA-8 devices without and with AALs (OAm). e, The statistics of power conversion efficiency

7 (PCE) distribution for 100 devices (50 pristine devices and 50 devices with AALs (OAm)). 

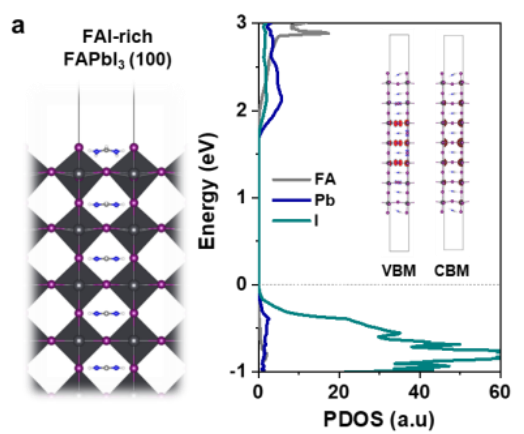

b

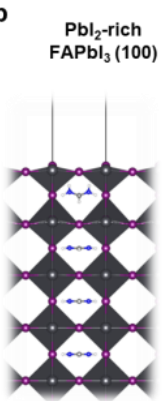

8 minimum $(C B M)$, and the trap states.
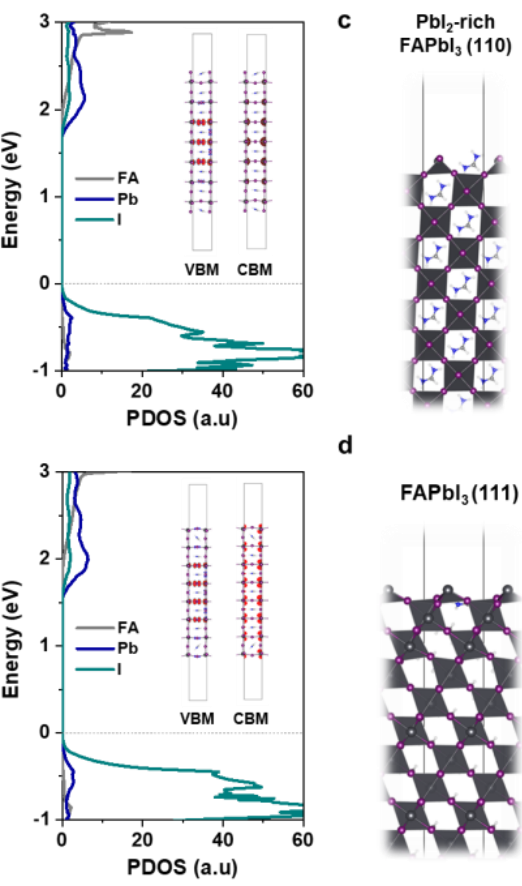

d

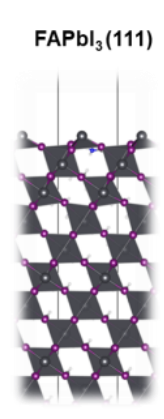

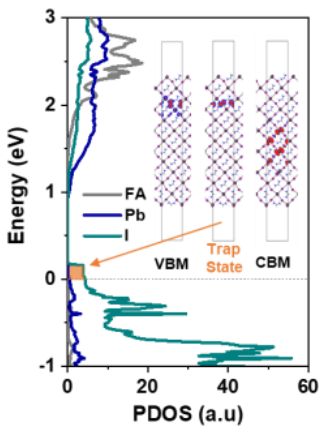
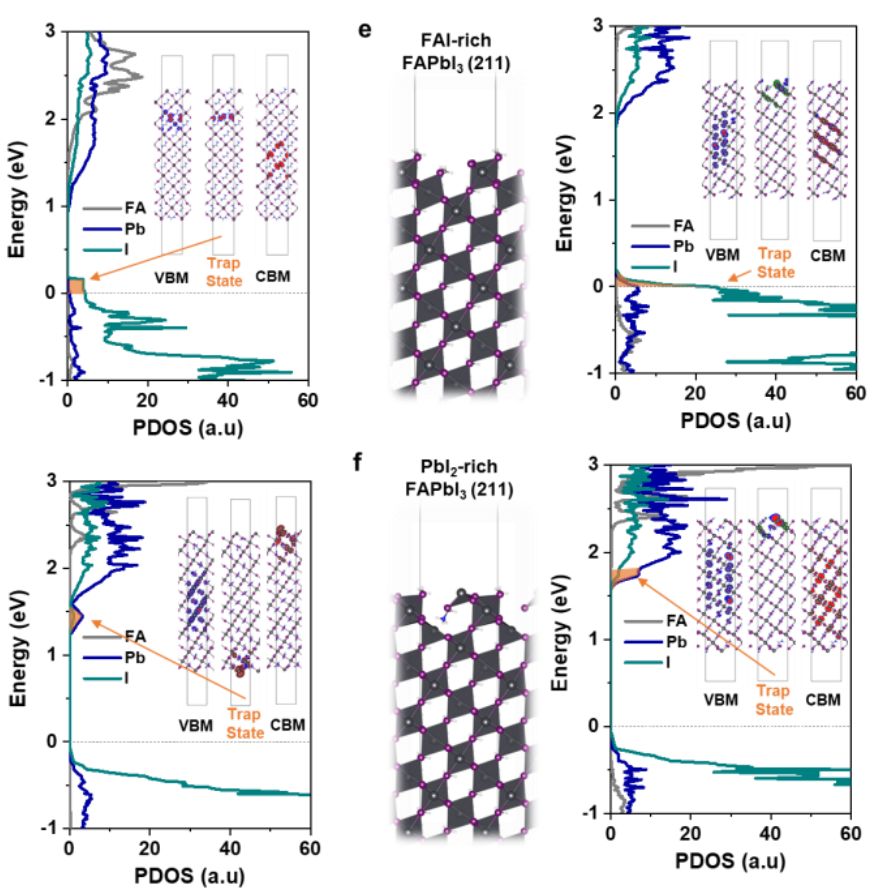

Figure 4. DFT calculation. Optimized crystal structures and projected density of states of cubicphase $\mathrm{FAPbI}_{3}$ slabs with surface facets: FAI-rich and PbI2-rich (100) (a and b), PbI2-rich (110) (c), (111) (d), and FAI-rich and PbI2-rich (211) (e and $\boldsymbol{f}$ ) calculated by density functional theory.

The insets are the charge densities for the valence band maximum (VBM), the conduction band 
a

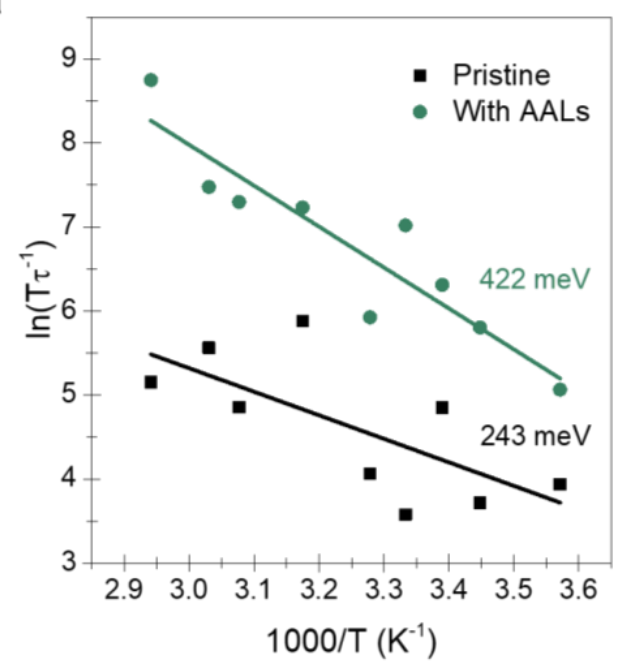

b

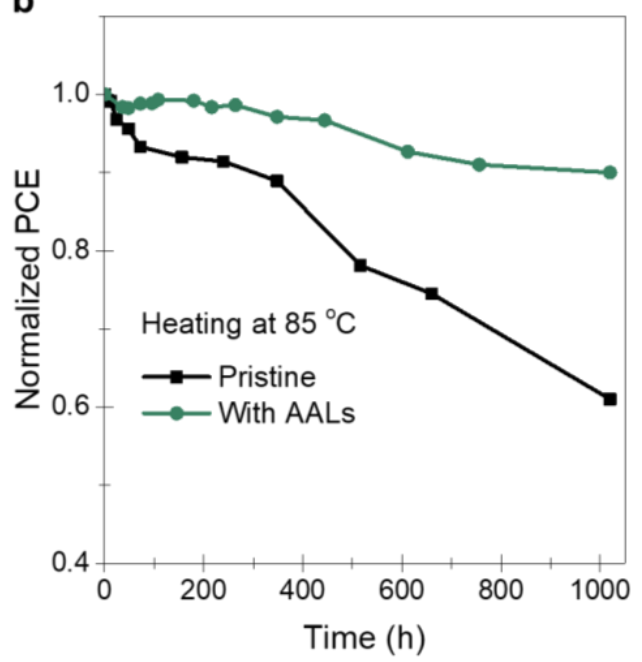

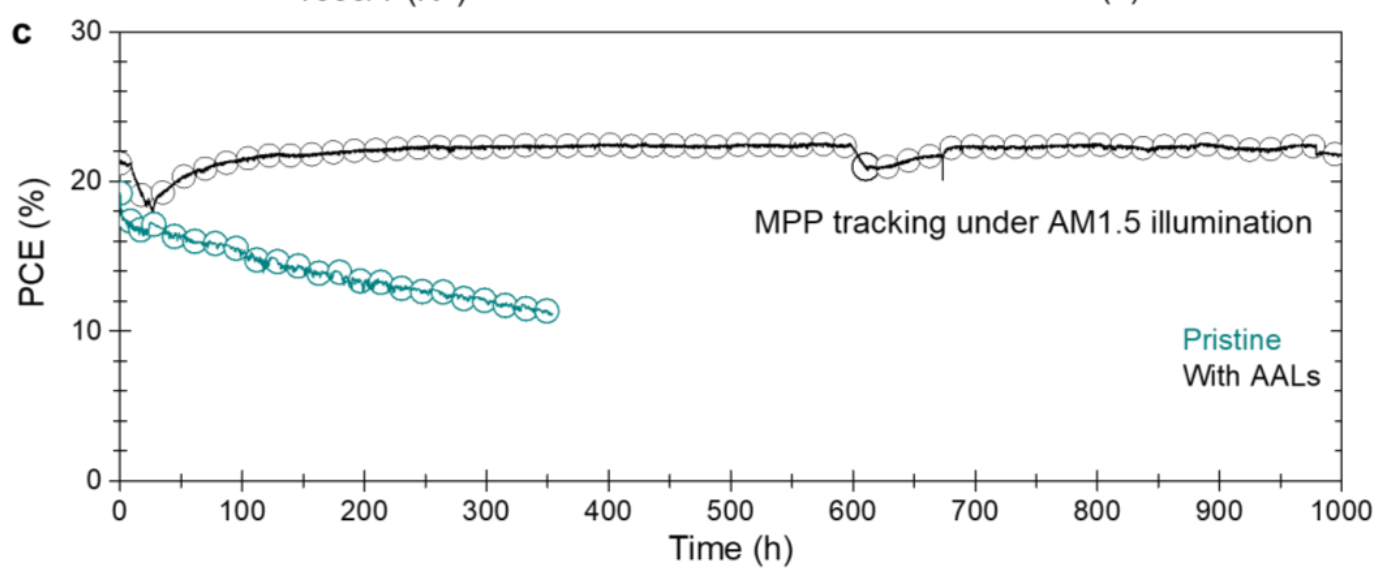

15 Figure 5. Ion migration and long-term device stability. a, Arrhenius plots of the temperature

16 dependence of $\tau^{-1} T$. Note $\tau^{-1}$ is the decay rate $\left(s^{-1}\right)$. $\boldsymbol{b}$, Thermal stability of devices at a fixed temperature of $85^{\circ} \mathrm{C}$ in a nitrogen atmosphere. $\boldsymbol{c}, 1000$ hours of continuous maximum power point

18 (MPP) tracking for pristine CsFAMA-8 device and the CsFAMA-8 device with AALs (OAm) under

19 constant simulated solar illumination $\left(100 \mathrm{~mW} \mathrm{~cm}^{-2}\right)$ in a nitrogen atmosphere with a $420-\mathrm{nm}$ 20 cutoff $U V$ filter. 
Managing grains and interfaces via ligand anchoring enables $22.3 \%$ certified-

23 efficiency inverted perovskite solar cells

24 Xiaopeng Zheng ${ }^{1, \uparrow}$, Yi $\mathrm{Hou}^{2, \dagger}$, Chunxiong $\mathrm{Bao}^{3}$, Jun Yin ${ }^{1}$, Fanglong Yuan ${ }^{2,4}$, Ziru Huang ${ }^{2}$, Kepeng

25 Song ${ }^{1}$, Jiakai Liu ${ }^{1}$, Joel Troughton ${ }^{1}$, Nicola Gasparini ${ }^{1}$, Chun Zhou ${ }^{2}$, Yuanbao Lin ${ }^{1}$, Dingjiang

$26 \mathrm{Xue}^{2}$, Bin Chen ${ }^{2}$, Andrew K. Johnston ${ }^{2}$, Nini $\mathrm{Wei}^{5}$, Mohamed Nejib Hedhili ${ }^{5}$, Mingyang Wei ${ }^{2}$,

27 Abdullah Y. Alsalloum ${ }^{1}$, Partha Maity ${ }^{1}$, Bekir Turedi ${ }^{1}$, Chen Yang ${ }^{1}$, Derya Baran ${ }^{1}$, Thomas D.

28 Anthopoulos ${ }^{1}$, Yu Han ${ }^{1}$, Zheng-Hong Lu ${ }^{4}$, Omar F. Mohammed ${ }^{1}$, Feng Gao ${ }^{3}$, Edward H. Sargent ${ }^{2}$,

$29 *$, and Osman M. Bakr ${ }^{1, *}$

$30{ }^{1}$ Division of Physical Sciences and Engineering, King Abdullah University of Science and

31 Technology (KAUST), Thuwal 23955-6900, Kingdom of Saudi Arabia.

$32{ }^{2}$ Department of Electrical and Computer Engineering, University of Toronto, 35 St George Street,

33 Toronto, Ontario, M5S 3G4, Canada.

$34{ }^{3}$ Department of Physics, Chemistry and Biology (IFM), Linköping University, Linköping, 35 Sweden.

${ }^{4}$ Department of Materials Science and Engineering, University of Toronto, 184 College Street,

37 Toronto, Ontario M5S 3E4, Canada.

39 (KAUST), Thuwal 23955-6900, Saudi Arabia. 

Trap density measurements by thermal admittance spectroscopy (TAS)

Based on the capacitance spectra measured at different temperatures, the trap density distribution in energy $(E \omega)$ was calculated with the following relations:

$$
E_{\omega}=k T \ln \left(\frac{\omega_{0}}{\omega}\right)=k T \ln \left(\frac{2 \pi v_{0} T^{2}}{\omega}\right)
$$
where $V_{b i}$ is the built-in potential and $W$ is the depletion width ( $V_{b i}$ and $W$ are derived from capacitance-voltage measurements), $C$ is the capacitance measured at an angular frequency $\omega$ and temperature $T, k$ is the Boltzmann constant, and $\omega_{0}$ is the attempt-to-escape frequency at temperature of $T, v_{0}$ is a temperature-independent constant which can be obtained by fitting the relation of characteristic frequency with different $\mathrm{T}$ based on equation (2).

\section{Ion migration measurement}

The slower decay time constants $\tau$ which represent the ion distribution recovery time were used to deduce the activation energy of the ion migration according to the following equation:

$$
\ln \left(T \tau^{-1}\right)=C-\frac{E_{a}}{k T}
$$
the Boltzmann constant. 

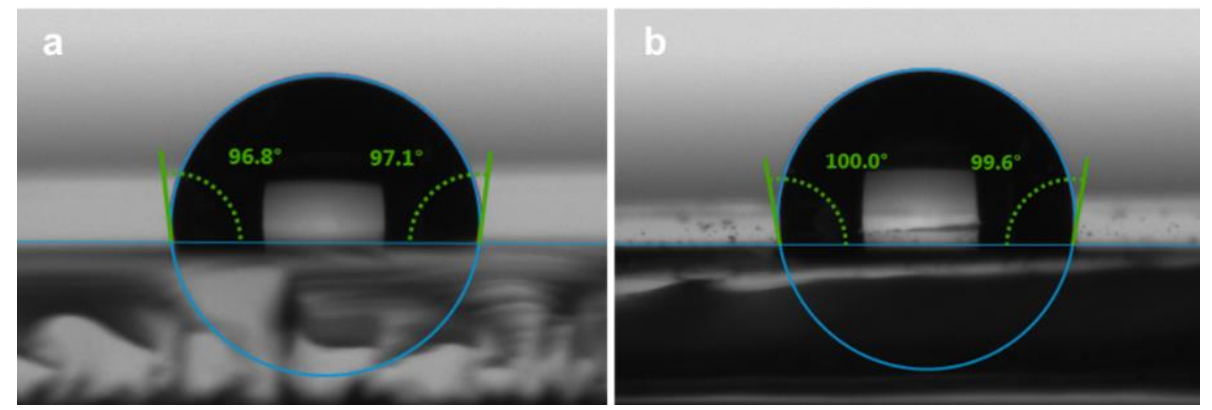

62 Supplementary Figure 1. a, b Water contact angles of the CsFAMA-8 film with 0.05 wt\% AALs 63 (OAm) and the CsFAMA-8 film with $0.2 \mathrm{wt} \%$ AALs (OAm), respectively. 


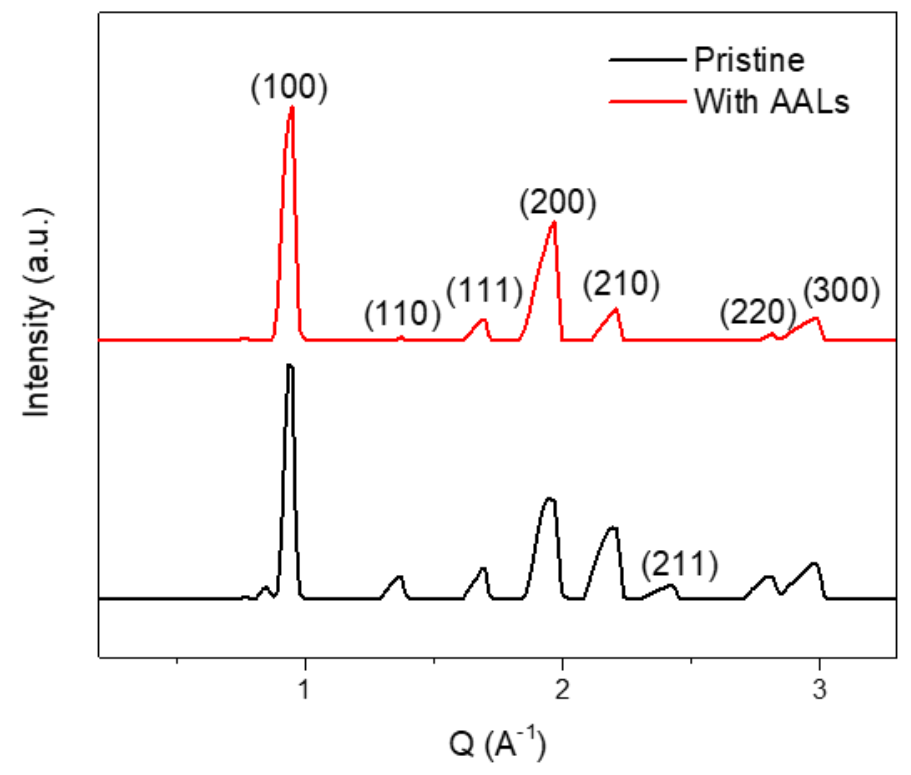

64

65 Supplementary Figure 2. Integrated intensity of GIWAXS data along $\mathrm{q}_{\mathrm{z}}$ for pristine CsFAMA-8 66 film and the CsFAMA-8 film with AALs (OAm). 


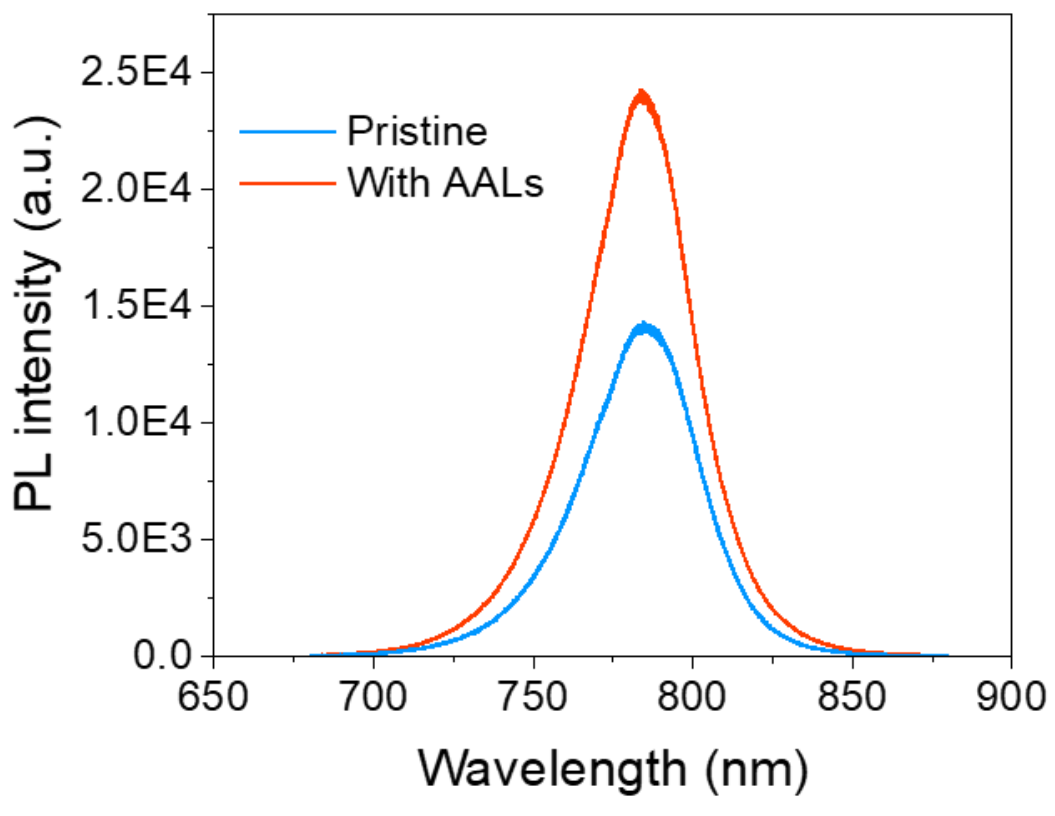

68 Supplementary Figure 3. Photoluminescence (PL) for pristine CsFAMA-8 film and the 69 CsFAMA-8 film with AALs (OAm). 


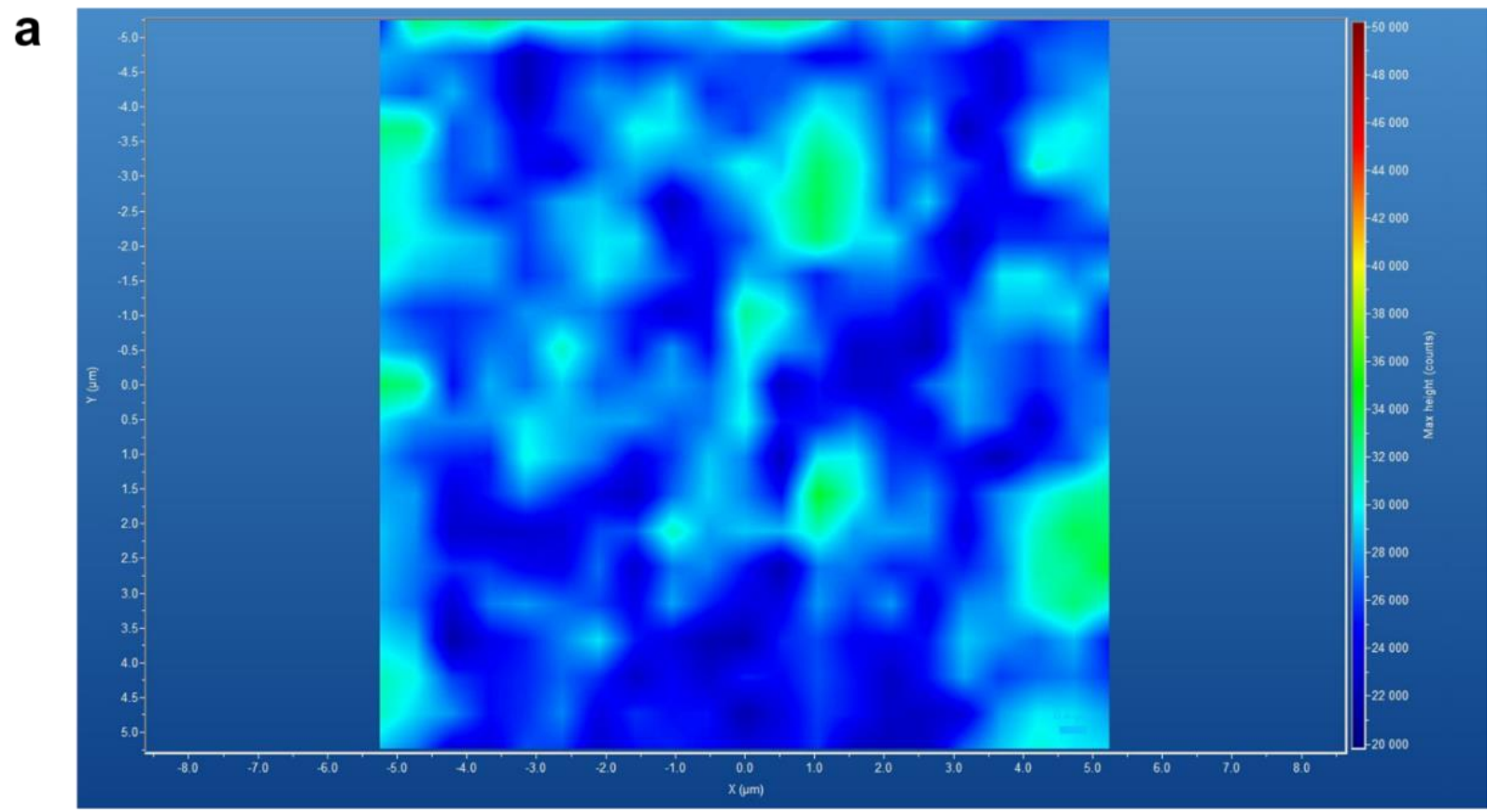

b

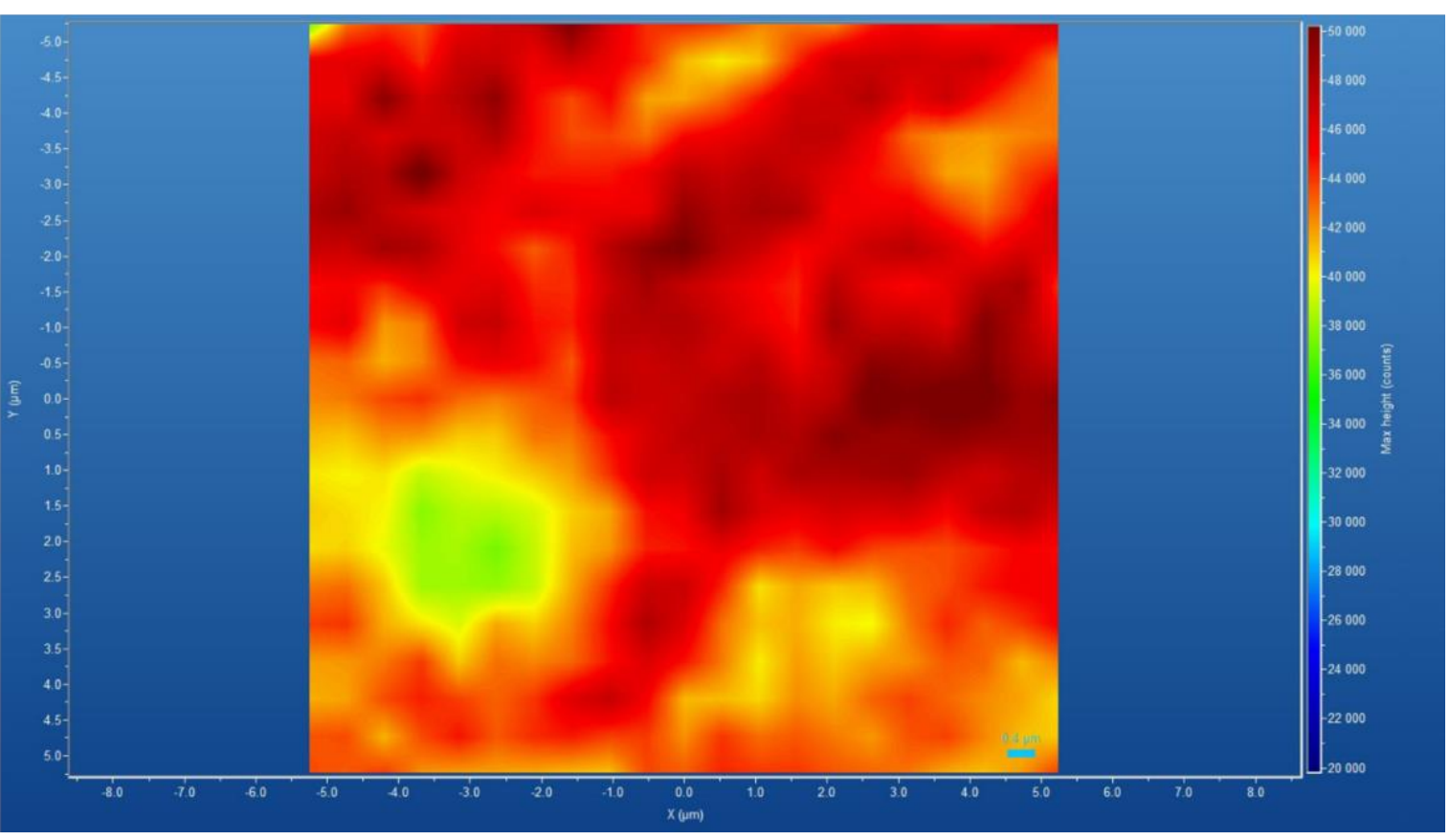

Supplementary Figure 4. a and b, Photoluminescence (PL) mapping for pristine CsFAMA-8 film

72 and the CsFAMA-8 film with AALs (OAm), respectively. 


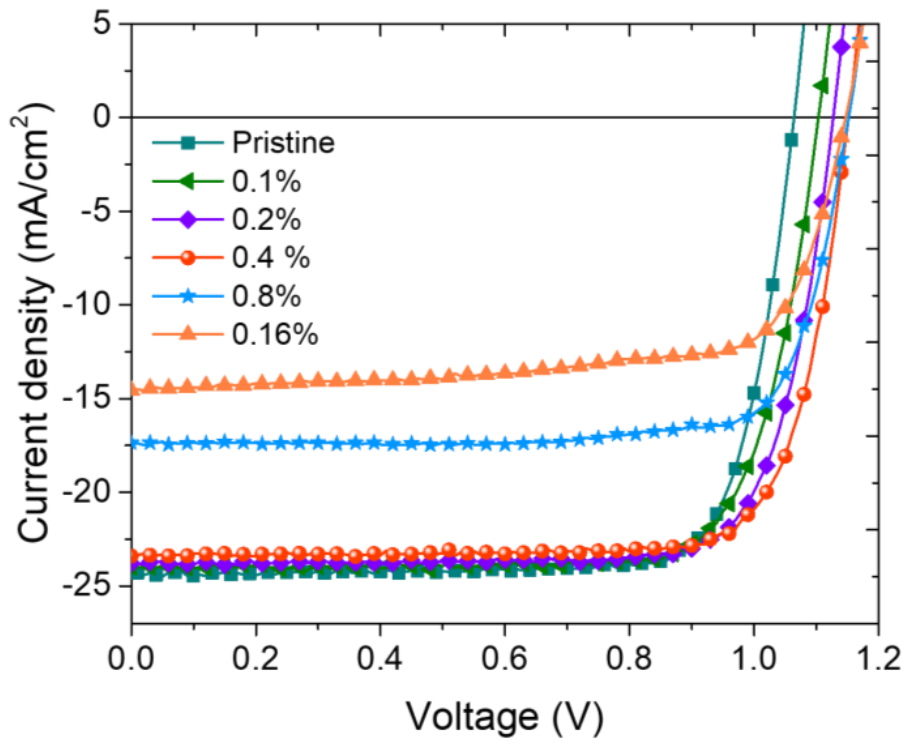

74 Supplementary Figure 5. PEA concentration-dependent current density-voltage $(J-V)$ 75 characteristics of CsFAMA-8 devices with PEA.

76 


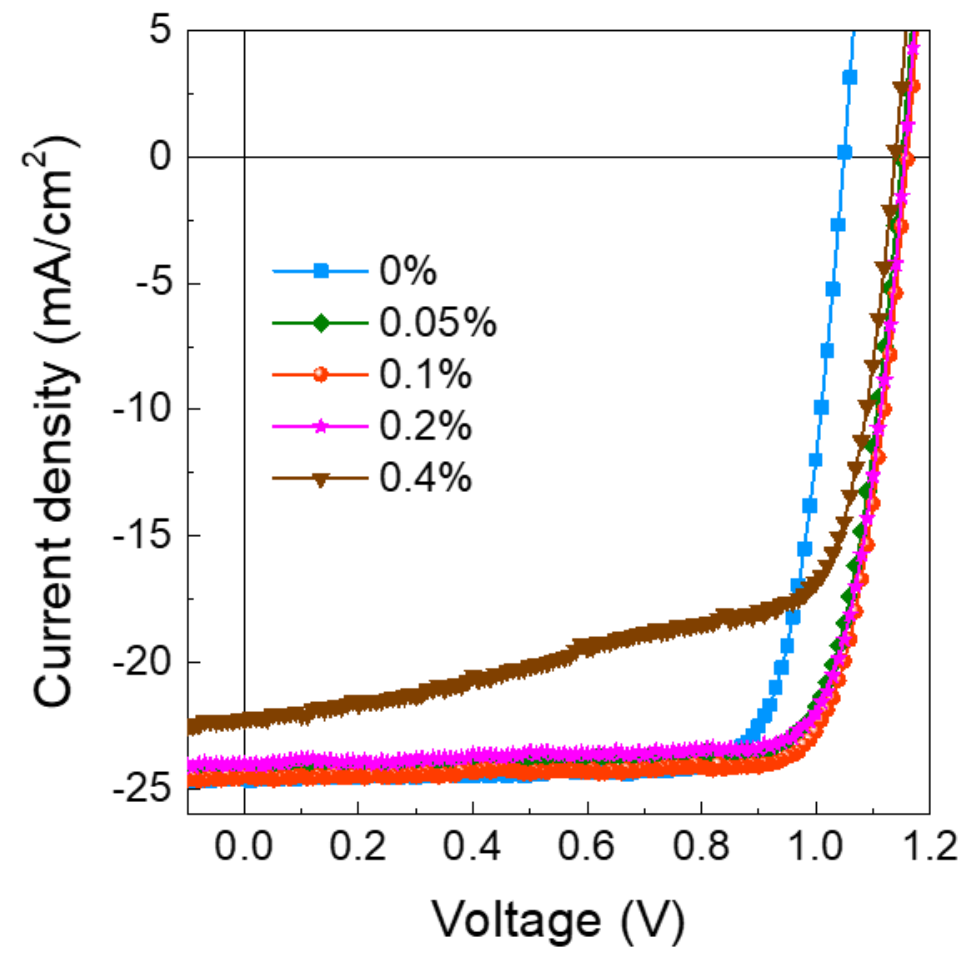

77

78 Supplementary Figure 6. $J-V$ characteristics of CsFAMA-8 devices with different AAL (OAm) 79 concentrations. 


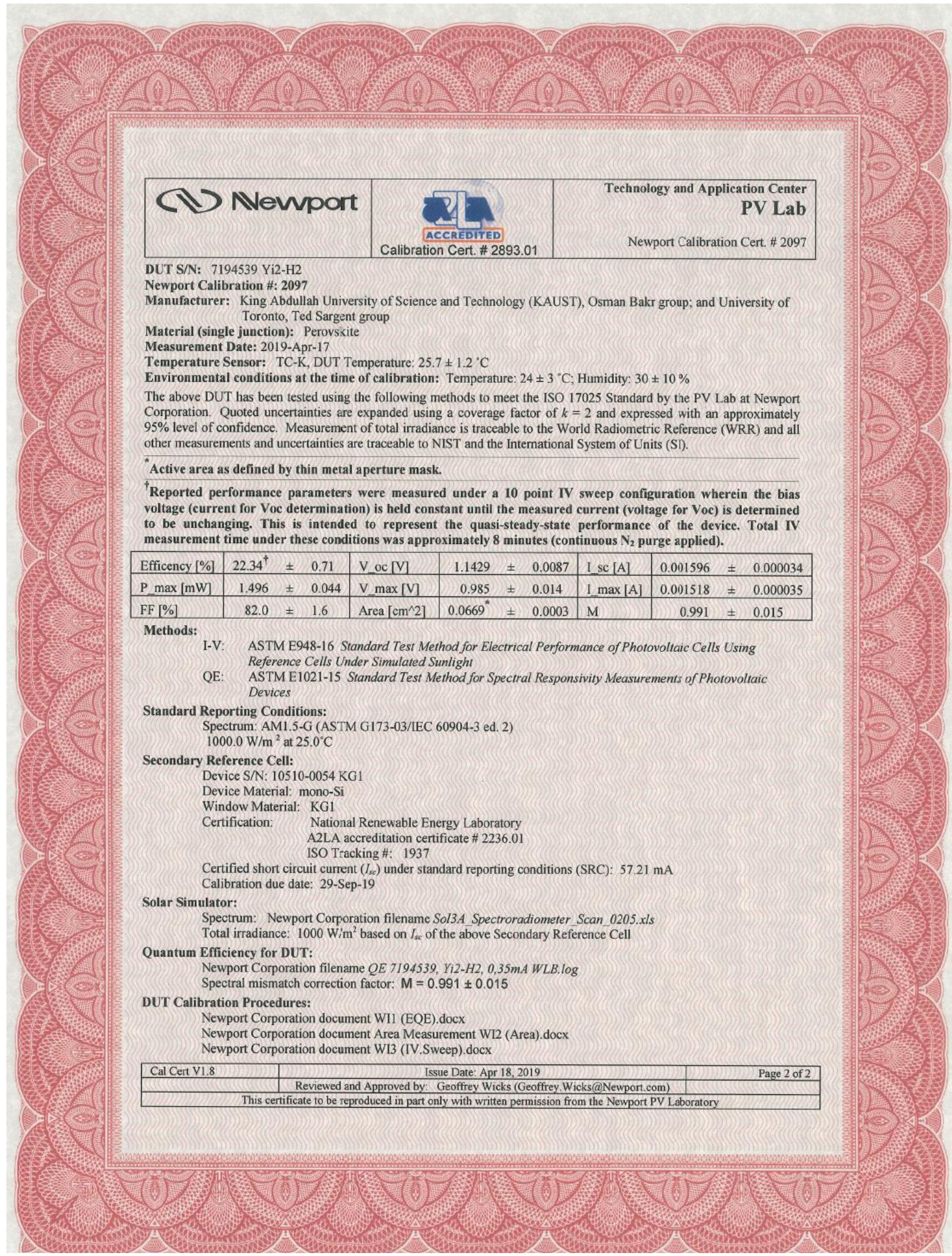


81 Supplementary Figure 7. Independent PCE certification of CsFAMA-8 inverted perovskite solar

82 cells with AALs by an accredited laboratory (Newport Photovoltaic Testing and Calibration

83 Laboratory in the U.S.), confirming a stabilized PCE of $22.34 \pm 0.71 \%$, which is a record certified

84 efficiency for inverted structured perovskite solar cells. 
a

\begin{tabular}{|r|r|}
\hline Newport & Technology and Application Center \\
PV Lab \\
Calibration Number 2097
\end{tabular}

IV Curve Cell \#7194539, Yi 2, H2

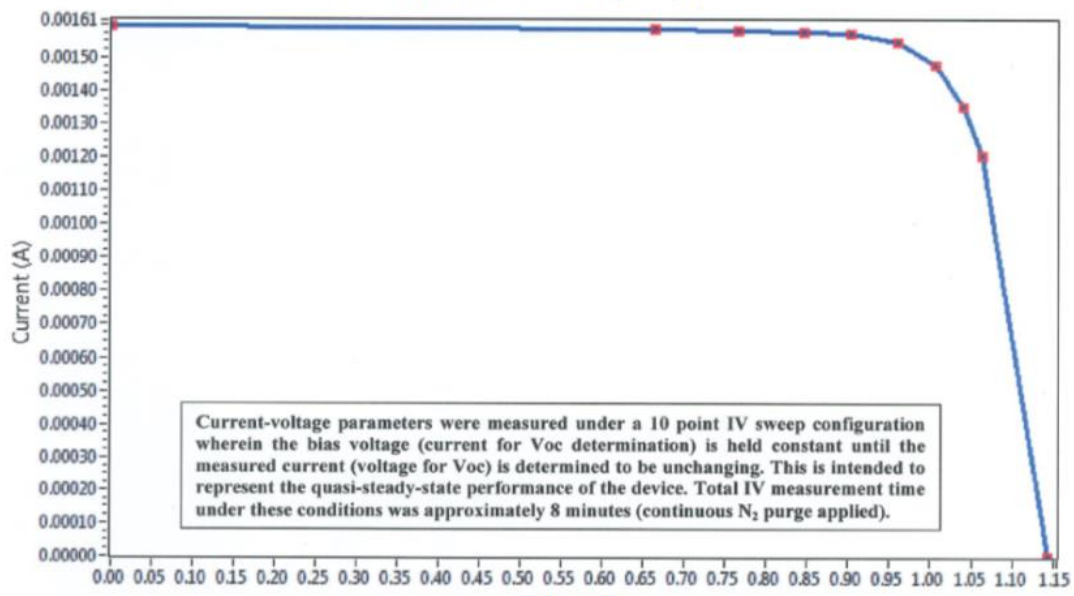

Voltage (V)

b

\begin{tabular}{|l|c|r|}
\hline Cal Cert Data V1_2 & Issue Date: Apr 18,2019 & Page 1of 3 \\
\hline & $\begin{array}{c}\text { Reviewed and Approved by: Geoffrey Wicks } \\
\text { Geoffrey.Wicks@Newport.com }\end{array}$ & \\
\hline
\end{tabular}

\begin{tabular}{|r|r|}
\hline Nevmport & Technology and Application Center \\
PV Lab \\
Calibration Number 2097
\end{tabular}

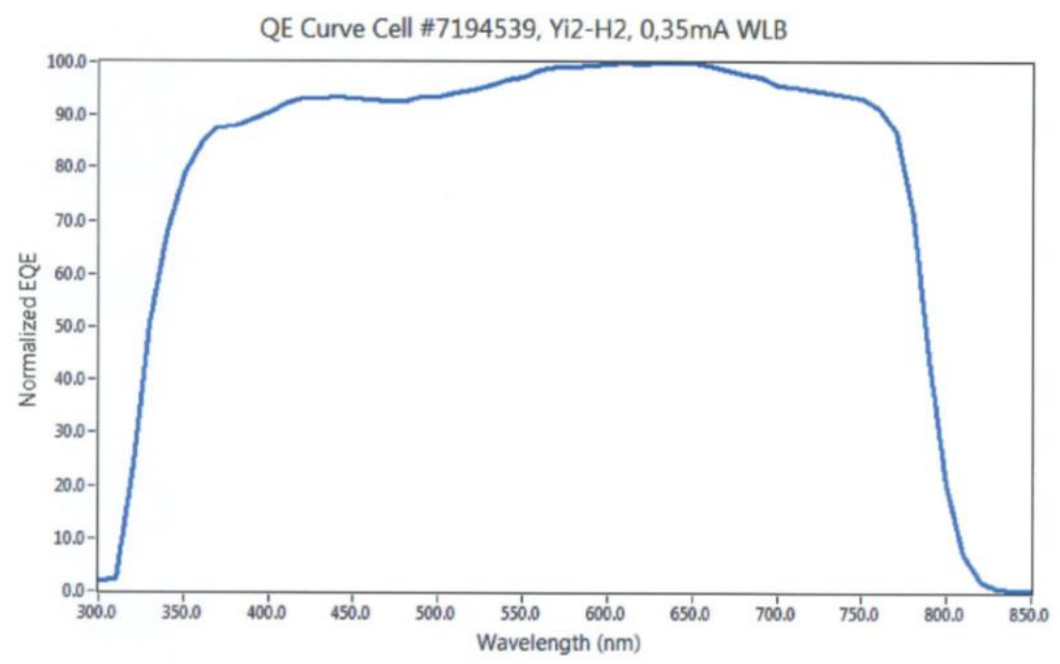

\begin{tabular}{|l|c|r|}
\hline Cal Cert Data V1_2 & Issue Date: Apr 18,2019 & Page 2of 3 \\
\hline & $\begin{array}{c}\text { Reviewed and Approved by: Geoffrey Wicks } \\
\text { Geoffrey.Wicks@Newport.com }\end{array}$ & \\
\hline
\end{tabular}


86 Supplementary Figure 8. a, Quasi-steady-state (QSS) $J$ - $V$ characteristics of CsFAMA-8 inverted

87 perovskite solar cells with AALs measured by Newport PV Lab under a 10 points I-V sweep

88 protocol. 10 voltage points were collected in QSS measurement, and each bias voltage is applied

89 and held until the measured current is determined to be unchanging. b, EQE of CsFAMA-8

90 inverted perovskite solar cells with AALs measured by Newport PV Lab. 

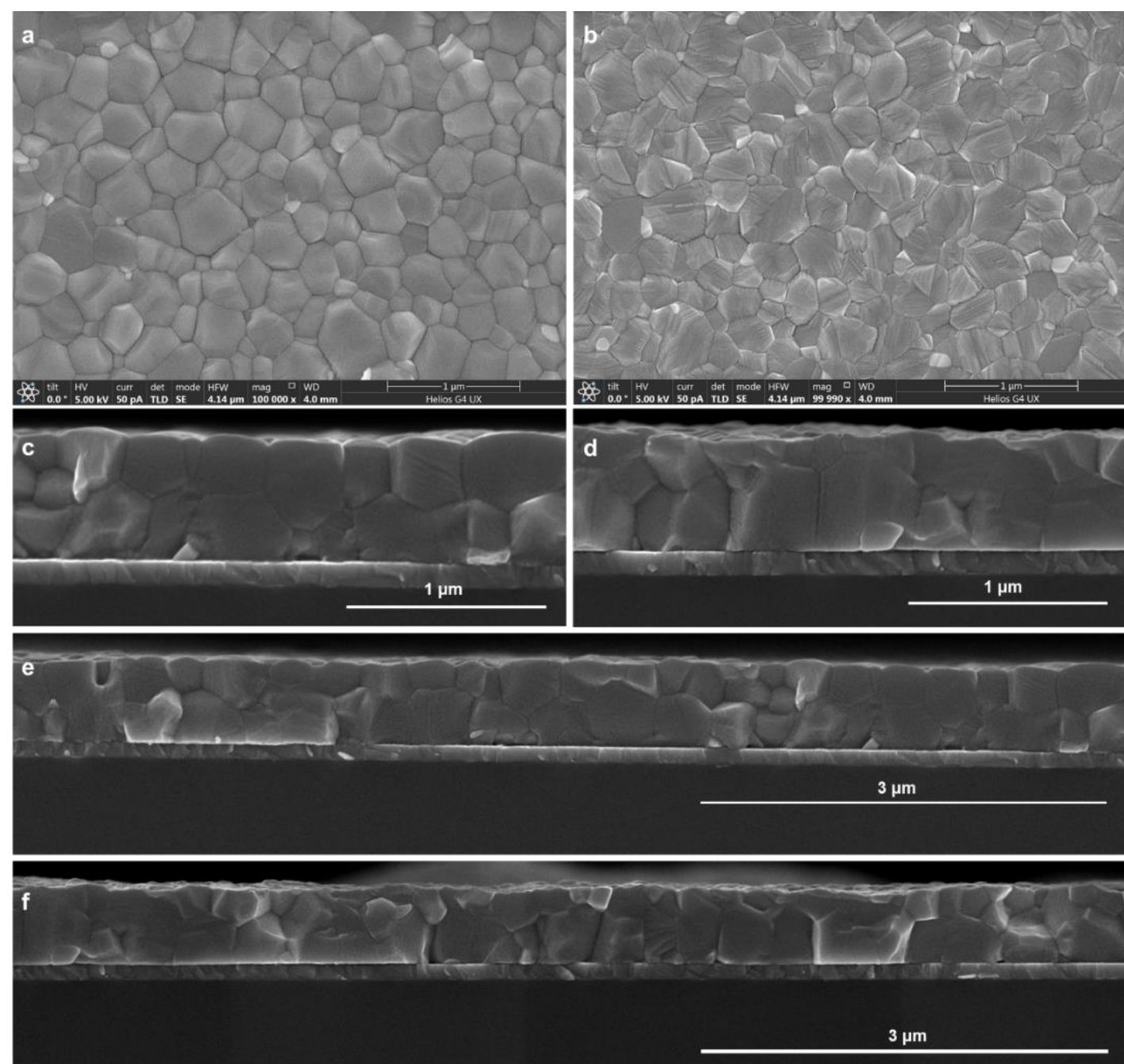

92 Supplementary Figure 9. a and b, Top-view SEM images of the pristine CsFAMA-8 film and

93 the CsFAMA-8 film with AALs (OAm), respectively. $\mathbf{c}$ and d, Cross-sectional SEM images of the

94 pristine CsFAMA-8 film and the CsFAMA-8 film with AALs (OAm), respectively. e and f, Large-

95 scale cross-sectional SEM images of the pristine CsFAMA-8 film and the CsFAMA-8 film with

96 AALs (OAm), respectively. 

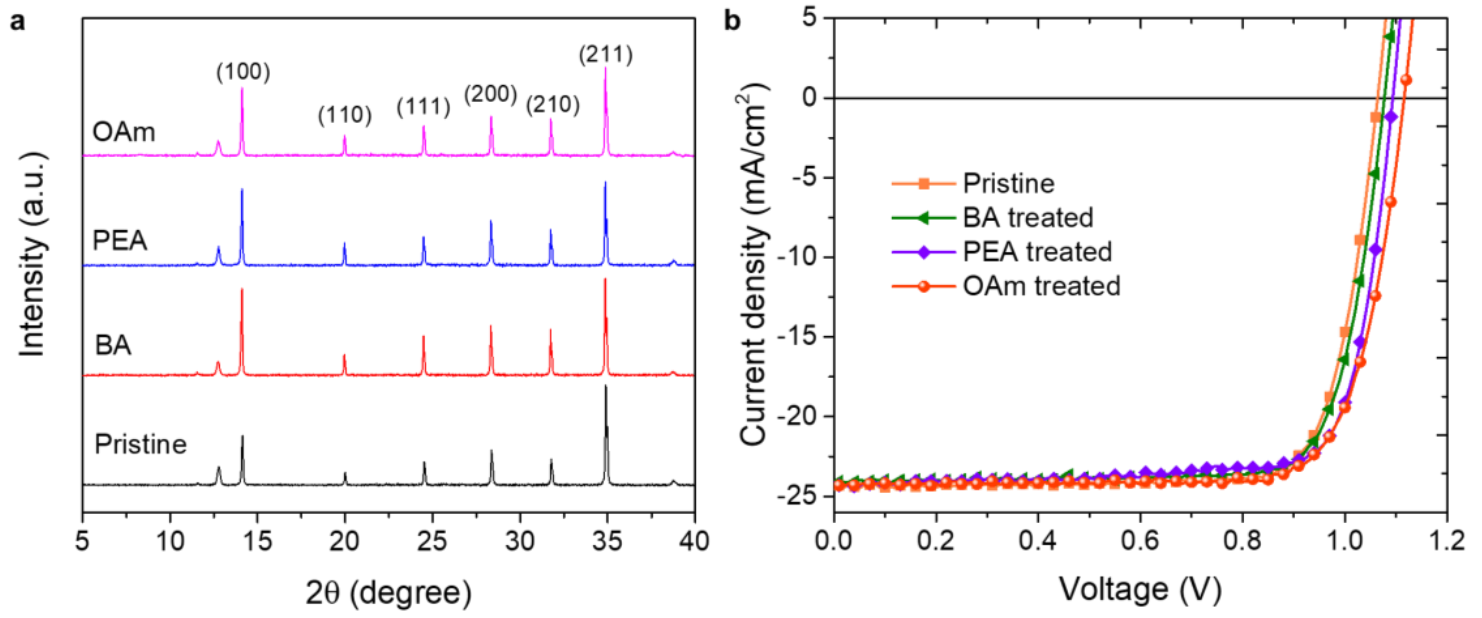

97

98 Supplementary Figure 10. a, XRD patterns of CsFAMA-8 perovskite films with different alkyl

99 chain length AAL (BA, PEA, and OAm) post-treatment. b, Current density-voltage $(J-V)$

100 characteristics of CsFAMA-8 devices with different alkyl chain length AAL (BA, PEA, and OAm)

101 post-treatment. 

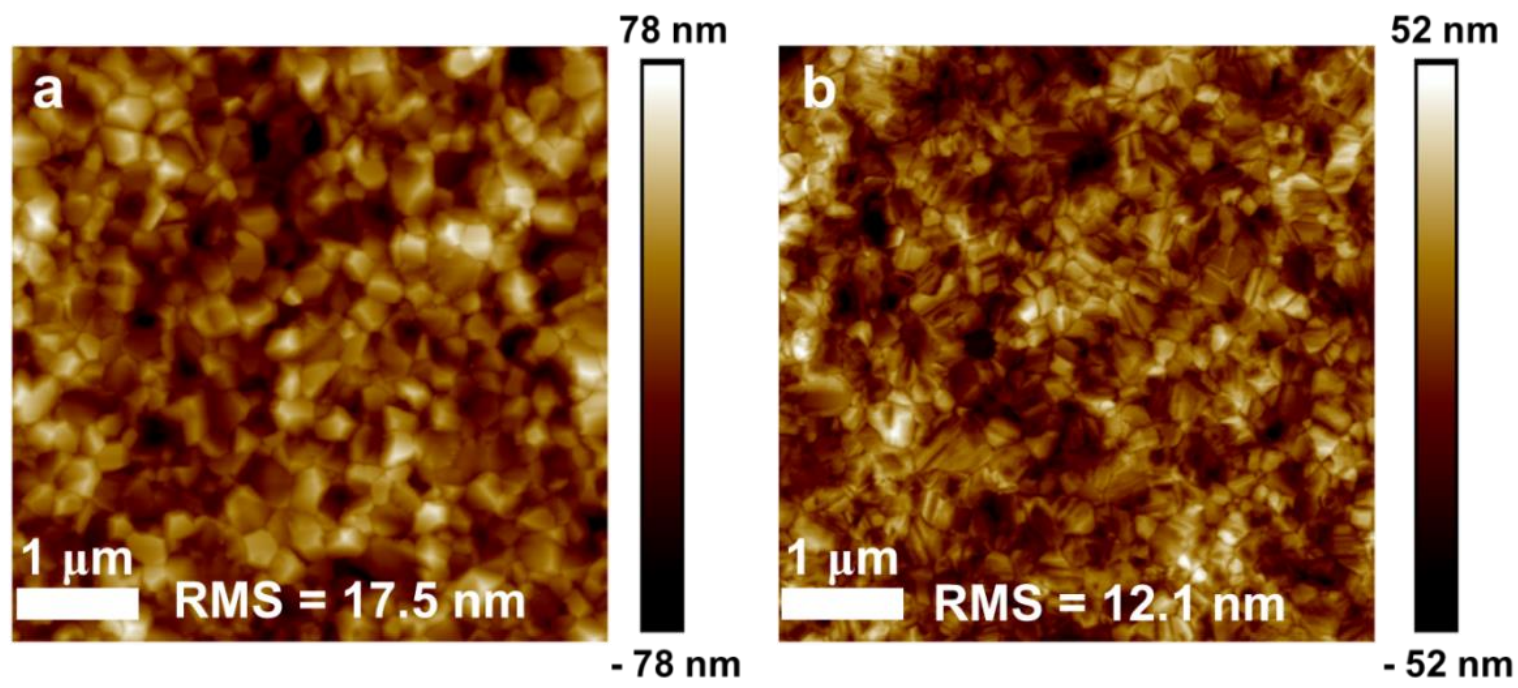

102

103 Supplementary Figure 11. a and b, Atomic force microscopy (AFM) images for pristine

104 CsFAMA-8 film and the CsFAMA-8 film with AALs (OAm), respectively. 

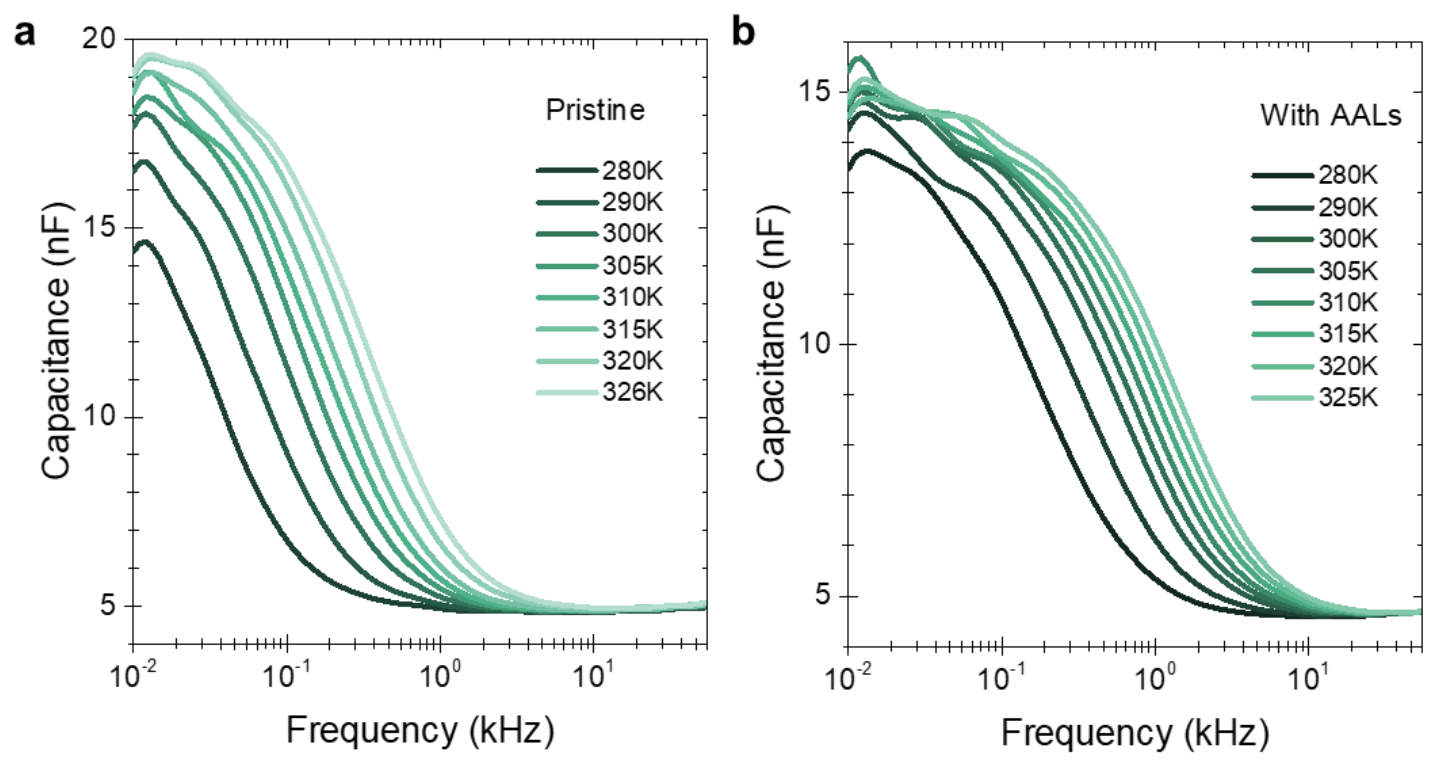

105

106 Supplementary Figure 12. a and b, Temperature dependence of $C-f$ plots for the pristine 107 CsFAMA-8 device and the CsFAMA-8 device with AALs (OAm), respectively. 


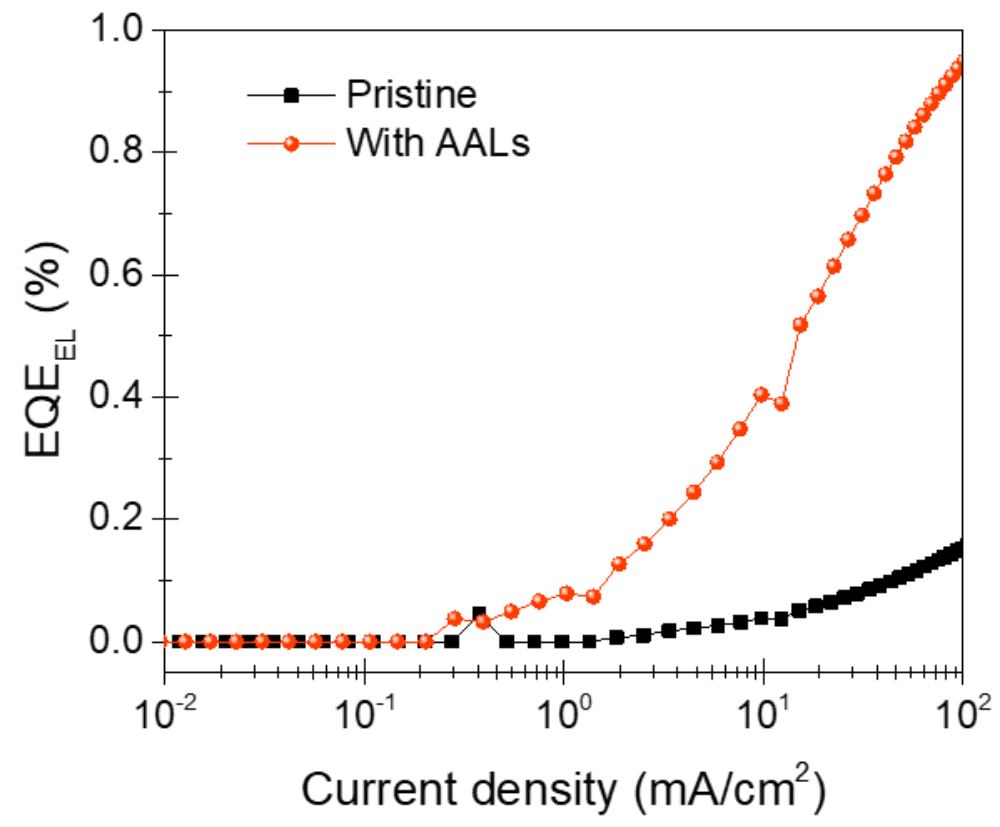

109

Supplementary Figure 13. EQE of the devices operated as LEDs. 


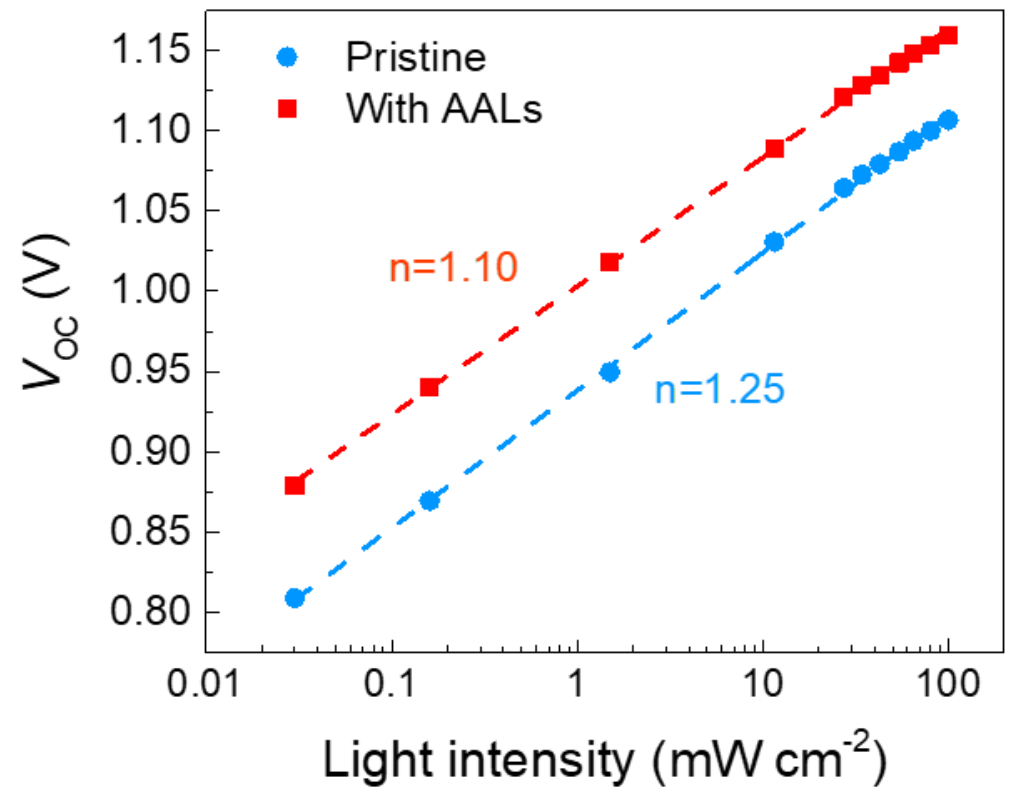

110

111 Supplementary Figure 14. The light intensity-dependent $V_{O C}$ for pristine CsFAMA-8 device and

112 the CsFAMA-8 device with AALs (OAm). 
a

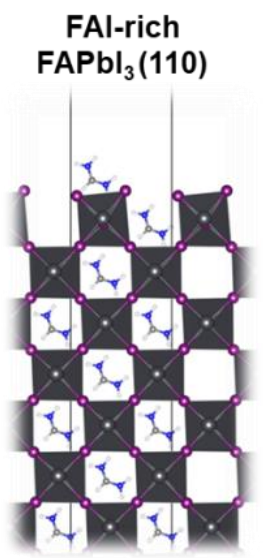

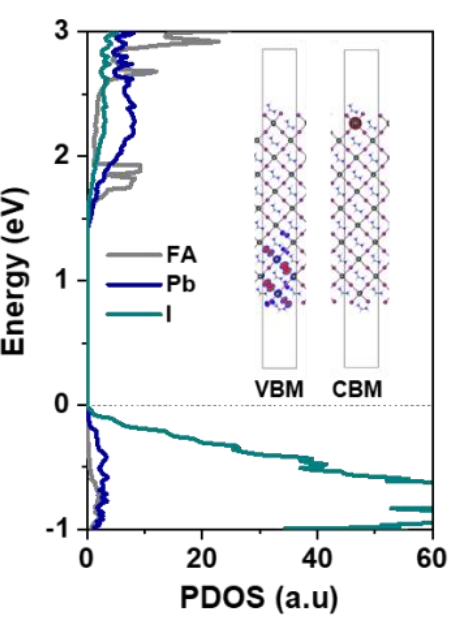

b
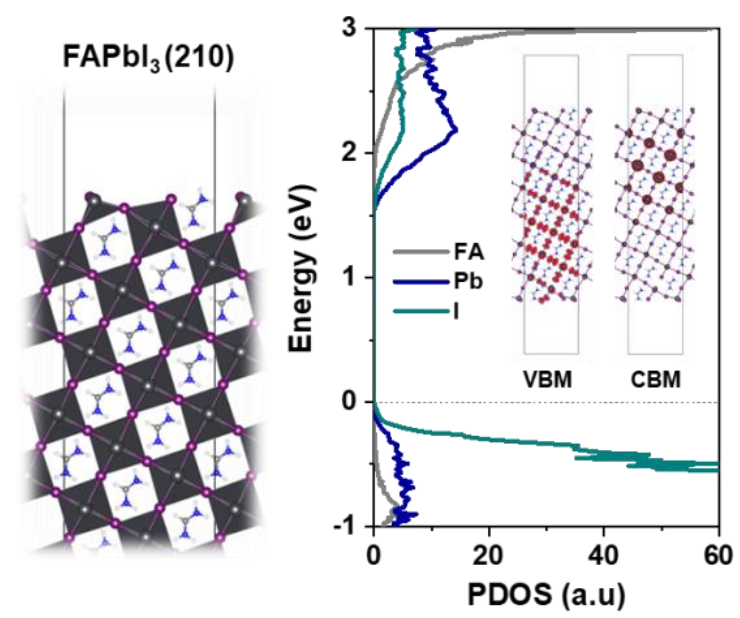

113

114 Supplementary Figure 15. Optimized crystal structures and projected density of states of cubic-

115 phase FAPbI3 slabs with surface facets FAI-rich (110) (a) and (210) (b). 
a

$\uparrow$ [h00] direction $\square$ Perovskite $\leadsto$ Short-chain AALs

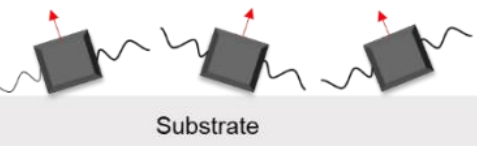

Nucleation

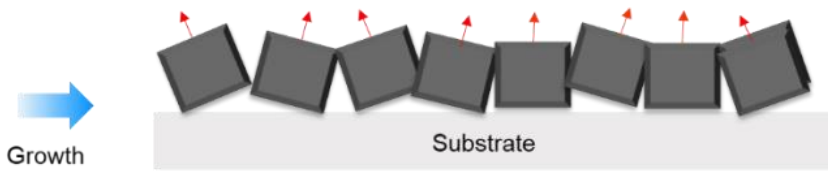

b

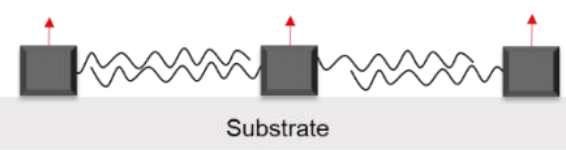

Nucleation
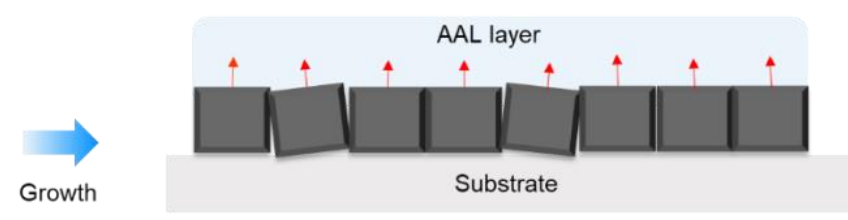

c

d

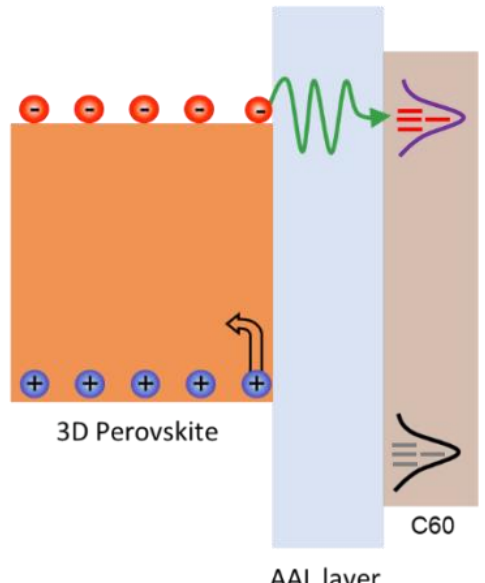

Perovskite

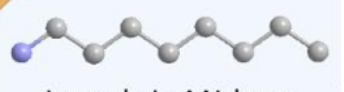

AAL layer

117 Supplementary Figure 16. A schematic illustration of the likely mechanisms of the efficiency enhancement for the devices with long chain AALs. a and b, Illustration of the influence of the

119 short-chain AALs and long-chain AALs on the crystallization of the perovskite films, respectively.

120 c and d, Schematic illustration of long chain AALs assembled on perovskite film surface and blocking the holes at the perovskite and $\mathrm{C}_{60}$ interfaces, respectively. 


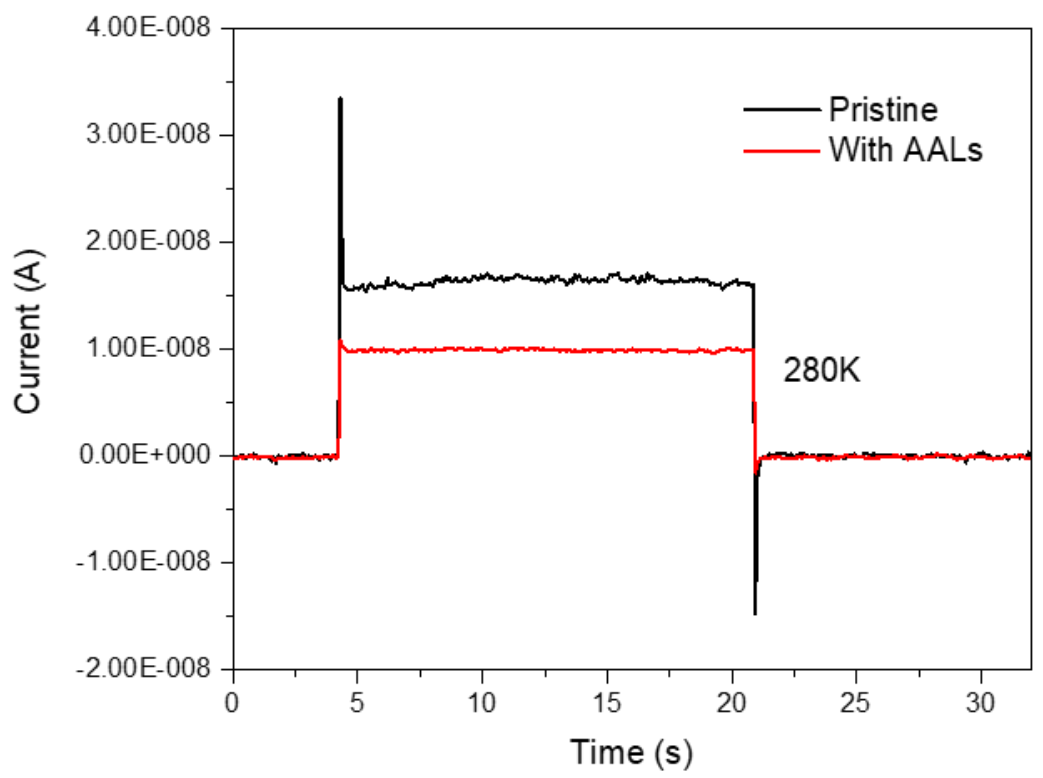

122

123 Supplementary Figure 17. A typical charging-discharging process for pristine CsFAMA-8 film 124 and the CsFAMA-8 film with AALs (OAm) measured at $280 \mathrm{~K}$. 

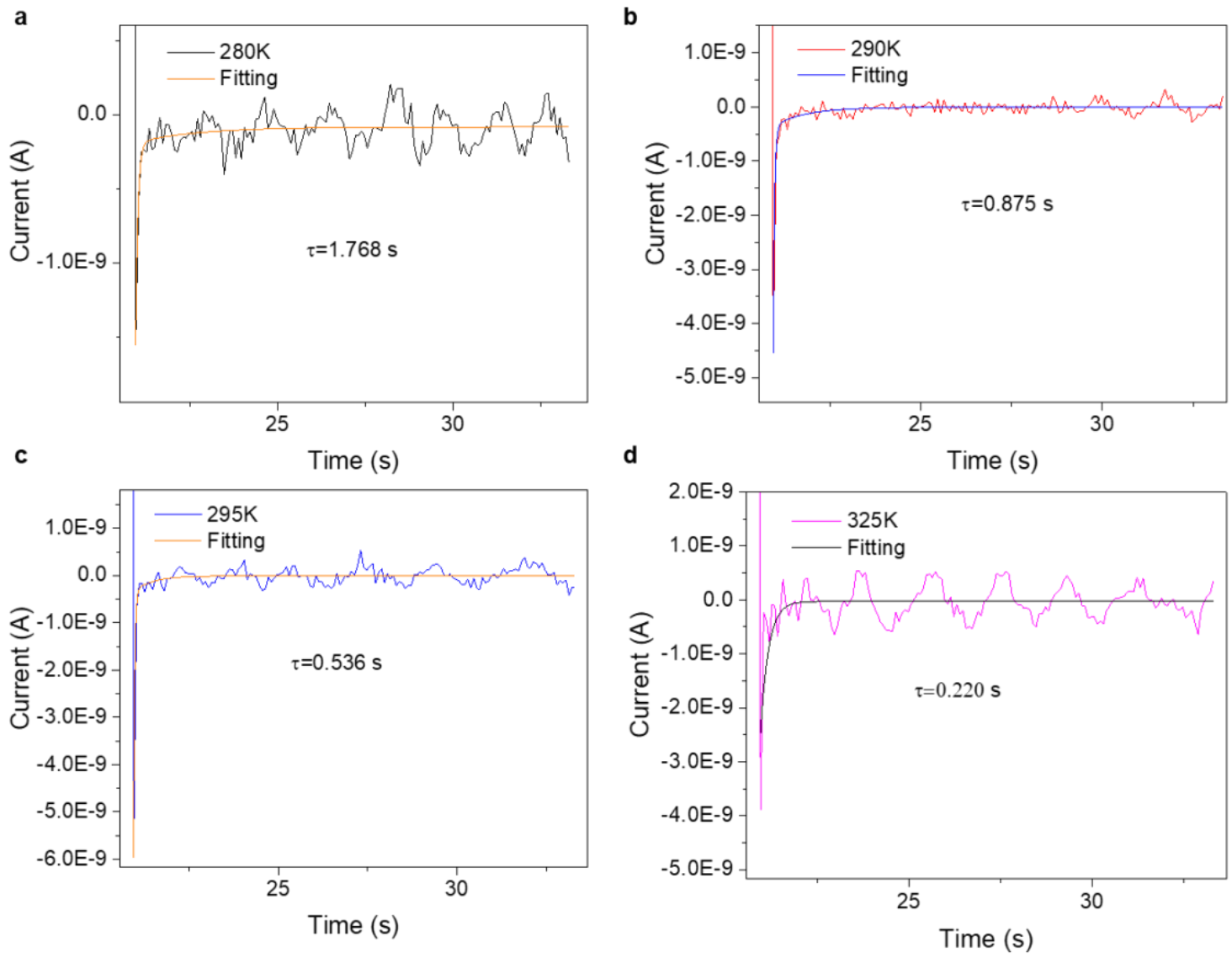

125

126 Supplementary Figure 18. Typical temperature-dependent transient response measurements for 127 CsFAMA-8 devices with AALs (OAm). 